Discrete ANALYSis, 2017:19, 41 pp.

www.discreteanalysisjournal.com

\title{
Ergodicity of the Liouville System Implies the Chowla Conjecture
}

\author{
Nikos Frantzikinakis \\ Received 18 September 2017; Published 12 December 2017
}

\begin{abstract}
The Chowla conjecture asserts that the values of the Liouville function form a normal sequence of plus and minus ones. Reinterpreted in the language of ergodic theory it asserts that the Liouville function is generic for the Bernoulli measure on the space of sequences with values plus or minus one. We show that these statements are implied by the much weaker hypothesis that the Liouville function is generic for an ergodic measure. We also give variants of this result related to a conjecture of Elliott on correlations of multiplicative functions with values on the unit circle. Our argument has an ergodic flavor and combines recent results in analytic number theory, finitistic and infinitary decomposition results involving uniformity seminorms, and qualitative equidistribution results on nilmanifolds.
\end{abstract}

Key words and phrases: Multiplicative functions, Liouville function, Möbius function, Chowla conjecture, Elliott conjecture, Sarnak conjecture, Gowers uniformity, inverse theorems.

\section{Introduction and main results}

\subsection{Introduction}

Let $\lambda: \mathbb{N} \rightarrow\{-1,1\}$ be the Liouville function which is defined to be 1 on integers with an even number of prime factors, counted with multiplicity, and -1 elsewhere. It is generally believed that the values of the Liouville function enjoy various randomness properties and one manifestation of this principle is an old conjecture of Chowla [5] which asserts that for all $\ell \in \mathbb{N}$ and all distinct $n_{1}, \ldots, n_{\ell} \in \mathbb{N}$ we have

$$
\lim _{M \rightarrow \infty} \frac{1}{M} \sum_{m=1}^{M} \lambda\left(m+n_{1}\right) \cdots \lambda\left(m+n_{\ell}\right)=0 .
$$




\section{NIKOS FRANTZIKINAKIS}

The conjecture is known to be true only for $\ell=1$; this case is elementarily equivalent to the prime number theorem. For $\ell=2$ and for all odd values of $\ell \in \mathbb{N}$, a variant involving logarithmic averages was recently established by Tao [41] and Tao, Teräväinen [43] respectively, and an averaged form of the Chowla conjecture was established by Matomäki, Radziwiłł, and Tao [35] using a recent breakthrough of Matomäki and Radziwiłł [34] concerning averages of bounded multiplicative functions on typical short intervals. For all $\ell \geq 2$ the conjecture remains open for Cesàro averages and for all even $\ell \geq 4$ it remains open for logarithmic averages. It is a consequence of the previous results that all size three sign patterns are taken by consecutive values of $\lambda$ with positive lower density [36] (and in fact with logarithmic density $1 / 8$ [43]) and all size four sign patterns are taken with positive lower density [43]. Similar results are not known for patterns of longer size and in fact out of the $2^{\ell}$ possible size $\ell$ sign patterns only $\ell+5$ of them are known to be taken by consecutive values of $\lambda$ with positive lower density [36] (the Chowla conjecture predicts that all $2^{\ell}$ patterns are taken and each one with density $2^{-\ell}$ ).

We can reinterpret the Chowla conjecture in the language of ergodic theory, hoping that this offers some appreciable advantage (a point of view also taken for example in [1,38]). Assuming for the moment that the limit on the left hand side of (1) exists for all $\ell \in \mathbb{N}$ and $n_{1}, \ldots, n_{\ell} \in \mathbb{N}$, we introduce in a natural way a dynamical system (see Proposition 2.3), which we call the "Liouville system". The Chowla conjecture implies that this system is a Bernoulli system, but up to now, randomness properties of the Liouville system that are much weaker than independence remain elusive. For instance, it is not known whether this system is of positive entropy, weakly mixing, or even ergodic. We prove that the much weaker hypothesis of ergodicity implies that the Liouville system is Bernoulli and implies the Chowla conjecture. This can be stated informally as follows (see Section 1.2.1 for the formal statements of our main results):

\section{Theorem. If the Liouville system is ergodic, then the Chowla conjecture is satisfied.}

Thinking of $\lambda$ as a point on the sequence space $\{-1,1\}^{\mathbb{N}}$, we can reformulate this result using notation from [18, Definition 3.4] as follows: If the Liouville function is generic for an ergodic measure on the sequence space, then the Chowla conjecture is satisfied.

An implicit assumption made in these statements is that the Liouville function admits correlations for Cesàro averages on the integers. In Section 1.2.1 we give results that do not depend on such strong hypothesis; we work with sequences of intervals with left end equal to 1 along which the Liouville function admits correlations for logarithmic averages (such sequences are guaranteed to exist), and Theorem 1.1 states that ergodicity of the corresponding measure preserving system implies that the Chowla conjecture holds for logarithmic averages on the same sequence of intervals. We deduce in Corollary 1.2 ergodicity conditions which imply that the Chowla conjecture holds for logarithmic averages taken on the full sequence of integers.

Three main ingredients enter the proof of Theorem 1.1:

1. A recent result of Tao (see Theorem 1.7) enables to reduce the Chowla conjecture for logarithmic averages to a local uniformity property of the Liouville function (this is the only reason why some of our statements involve logarithmic averages). Our goal then becomes to prove this uniformity property (stated in Theorem 1.6).

2. An inverse theorem for local uniformity seminorms, which takes a particularly useful form for ergodic sequences (see Theorem 4.1). In order to prove it we use both infinitary and finitary 
decomposition results (see Propositions 4.4 and 4.6). The former is proved via an ergodic inverse theorem of Host and Kra [28], and the latter via a finitistic inverse theorem of Green, Tao, and Ziegler [26]. The ergodicity of the sequence is essential; without this assumption we are led to conditions that we are unable to verify for the Liouville function.

3. An asymptotic orthogonality property of the Liouville function with nilsequences taken on typical short intervals (see Proposition 5.1); this is needed in order to verify that the aforementioned inverse theorem is applicable to the Liouville function. For Abelian nilsequences the orthogonality property follows from recent work of Matomäki, Radziwiłł, and Tao (see Proposition 2.10). For general nilsequences additional tools are needed; the heart of the argument is a result of purely dynamical context (see Proposition 5.6) and the only extra number-theoretic input needed is the orthogonality criterion of Lemma 5.5.

Our argument also works for the Möbius function; hence, ergodicity of the Möbius function implies a related Chowla-type result, and as a consequence, it also implies a conjecture of Sarnak [38, 39] stating that the Möbius function is uncorrelated with any bounded deterministic sequence. Moreover, our argument shows that every ergodic strongly aperiodic multiplicative function (see Definition 2.9) is locally uniform (see Theorem 1.6). This last property implies an Elliott-type result for this class of multiplicative functions (see Theorem 1.4) which in turn implies non-correlation with any bounded deterministic sequence.

\subsection{Main results}

In this subsection we give the precise statements of our main results, modulo notation that appears in the next section. We let $[N]=\{1, \ldots, N\}$.

\subsubsection{Ergodicity and Chowla's conjecture}

Our main result is the following (the notions used are explained in Section 2):

Theorem 1.1. Let $N_{k} \rightarrow \infty$ be a sequence of integers and $\mathbf{I}=\left(\left[N_{k}\right]\right)_{k \in \mathbb{N}}$. If the Liouville or the Möbius function is ergodic for logarithmic averages on $\mathbf{I}$, then it satisfies the Chowla conjecture for logarithmic averages on $\mathbf{I}$.

Remarks. - Since for every $\ell \in \mathbb{N}$ each size $\ell$ sign pattern is expected to be taken by consecutive values of $\lambda$, we cannot substitute the intervals $\left[N_{k}\right]$ with arbitrary intervals that do not start at 1 and have lengths increasing to infinity. The same comment applies to the results of the next subsection.

- We stress that if we assume ergodicity of the Liouville system for Cesàro (instead of logarithmic) averages on $\mathbf{I}$, our argument does not allow to deduce that the Chowla conjecture is satisfied for Cesàro averages on $\mathbf{I}$.

Since for every $a \in \ell^{\infty}(\mathbb{N})$ and $\mathbf{I}=\left(\left[N_{k}\right]\right)_{k \in \mathbb{N}}, N_{k} \rightarrow \infty$, there exists a subsequence $\mathbf{I}^{\prime}$ of $\mathbf{I}$ on which the sequence $a$ admits correlations, we deduce from Theorem 1.1 the following: 


\section{NIKOS FRANTZIKINAKIS}

Corollary 1.2. Suppose that whenever the Liouville (or the Möbius) function admits correlations for logarithmic averages on a sequence of intervals $\mathbf{I}=\left(\left[N_{k}\right]\right)_{k \in \mathbb{N}}, N_{k} \rightarrow \infty$, the induced measure preserving system is ergodic. Then the Liouville (resp. the Möbius) function satisfies the Chowla conjecture for logarithmic averages on $([N])_{N \in \mathbb{N}}{ }^{1}$

Note that this result does not impose an assumption of existence of correlations for logarithmic averages of the Liouville function; it rather states that in order to verify the Chowla conjecture for logarithmic averages it suffices to work under the assumption that correlations exist on some sequence of intervals $\mathbf{I}=\left(\left[N_{k}\right]\right)_{k \in \mathbb{N}}, N_{k} \rightarrow \infty$, and then for any such sequence $\mathbf{I}$ verify that the induced measure preserving system is ergodic.

Since convergence of Cesàro averages on $\mathbf{I}=([N])_{N \in \mathbb{N}}$ implies convergence to the same limit of logarithmic averages on $\mathbf{I}$, we deduce the result stated in the introduction:

Corollary 1.3. If the Liouville or the Möbius function is ergodic for Cesàro averages on $\mathbf{I}=([N])_{N \in \mathbb{N}}$, then it satisfies the Chowla conjecture for Cesàro averages on $\mathbf{I}$.

Further analysis of structural properties of measure preserving systems naturally associated with the Liouville or the Möbius function appear in the recent article of the author and Host [15]. The direction taken in [15] is complementary to the one in this article and the techniques used very different.

\subsubsection{Ergodicity and Elliott's conjecture}

We give a variant of our main result which applies to correlations of arbitrary multiplicative functions with values on the unit circle. This relates to logarithmically averaged variants of conjectures made by Elliott in $[9,10]$.

Theorem 1.4. Let $f_{1} \in \mathcal{M}$ be a strongly aperiodic multiplicative function which is ergodic for logarithmic averages on $\mathbf{I}=\left(\left[N_{k}\right]\right)_{k \in \mathbb{N}}, N_{k} \rightarrow \infty$. Then for every $s \geq 2$ we have that

$$
\mathbb{E}_{m \in \mathbf{I}}^{\log } f_{1}\left(m+n_{1}\right) \cdots f_{s}\left(m+n_{s}\right)=0
$$

holds for all $f_{2}, \ldots, f_{s} \in \mathcal{M}$ and all distinct $n_{1}, \ldots, n_{s} \in \mathbb{N}$.

Elliott conjectured that the conclusion holds for Cesàro averages without the ergodicity assumption and under the weaker assumption of aperiodicity (which coincides with strong aperiodicity for real valued multiplicative functions), but in [35, Theorem B.1] it was shown that for complex valued multiplicative functions a stronger assumption is needed and strong aperiodicity seems to be the right one.

Specializing the previous result to the case $f_{1}=\cdots=f_{s}=f$ where $f$ is an aperiodic multiplicative function taking values plus or minus one only (aperiodicity implies strong aperiodicity in this case) we deduce the following:

Corollary 1.5. Let $f: \mathbb{N} \rightarrow\{-1,1\}$ be an aperiodic multiplicative function which admits correlations on $\mathbf{I}=\left(\left[N_{k}\right]\right)_{k \in \mathbb{N}}, N_{k} \rightarrow \infty$, for logarithmic averages. Then the Furstenberg system induced by $f$ and $\mathbf{I}$ for logarithmic averages is ergodic if and only if it is Bernoulli.

\footnotetext{
${ }^{1}$ It then follows from a recent result in [19] that there exists $M_{k} \rightarrow \infty$ such that $\lim _{k \rightarrow \infty} \mathbb{E}_{m \in\left[M_{k}\right]} \lambda\left(m+n_{1}\right) \cdots \lambda\left(m+n_{\ell}\right)=0$ for all $\ell \in \mathbb{N}$ and distinct $n_{1}, \ldots, n_{\ell} \in \mathbb{N}$.
} 


\subsubsection{Ergodicity and local uniformity}

The key step taken in this article in order to prove Theorem 1.1, is to establish local uniformity for the class of ergodic strongly aperiodic multiplicative functions. The precise statement is as follows (the notions used are explained in Section 2):

Theorem 1.6. Let $f \in \mathcal{M}$ be a strongly aperiodic multiplicative function which is ergodic for Cesàro (or logarithmic) averages on $\mathbf{I}=\left(\left[N_{k}\right]\right)_{k \in \mathbb{N}}, N_{k} \rightarrow \infty$. Then $\|f\|_{U^{s}(\mathbf{I})}=\|f\|_{U_{*}^{s}(\mathbf{I})}=0$ (corr. $\|f\|_{U_{\text {log }}^{s}(\mathbf{I})}=$ $\|f\|_{U_{*, l o g}^{s}(\mathbf{I})}=0$ ) for every $s \in \mathbb{N}$.

Remark. It is shown in [14] (and previously in [23, 25, 26] for the Möbius and the Liouville function) that if $f$ is an aperiodic multiplicative function, then for every $s \in \mathbb{N}$ we have $\lim _{N \rightarrow \infty}\left\|f_{N}\right\|_{U^{s}\left(\mathbb{Z}_{N}\right)}=0$ where $\|\cdot\|_{U^{s}\left(\mathbb{Z}_{N}\right)}$ are the Gowers uniformity norms and with $f_{N}$ we denote the periodic extension of $f \cdot \mathbf{1}_{[N]}$ to $\mathbb{Z}_{N}$. It should be stressed though, that when $\mathbf{I}=([N])_{N \in \mathbb{N}}$, the local uniformity condition $\|f\|_{U^{s}(\mathbf{I})}=0$ is strictly stronger and cannot be inferred from Gowers uniformity for any $s \geq 2$. For example, the (non-ergodic) sequence $a(n)=\sum_{k=1}^{\infty}(-1)^{k} \mathbf{1}_{\left[k^{2},(k+1)^{2}\right)}(n), n \in \mathbb{N}$, satisfies $\lim _{N \rightarrow \infty}\left\|a_{N}\right\|_{U^{s}\left(\mathbb{Z}_{N}\right)}=0$ for every $s \in \mathbb{N}$ where $a_{N}$ is defined as above, but $\|a\|_{U^{1}(\mathbf{I})}=1$. Moreover, the sequence $b(n)=\sum_{k=1}^{\infty}(-1)^{n+k} \mathbf{1}_{\left[k^{2},(k+1)^{2}\right)}(n)$, $n \in \mathbb{N}$, is ergodic for Cesàro averages on $\mathbf{I}$ and satisfies $\lim _{N \rightarrow \infty}\left\|b_{N}\right\|_{U^{s}\left(\mathbb{Z}_{N}\right)}=0$ for every $s \in \mathbb{N}$, but $\|b\|_{U^{2}(\mathbf{I})}=1$.

For a sketch of the proof of Theorem 1.6 see Section 5.1.

The link between Theorem 1.1 and Theorem 1.6 is given by the following result of Tao (it follows from [42, Theorem 1.8 and Remarks 1.9, 3.4]):

Theorem 1.7 (Tao [42]). Let $s \in \mathbb{N}, f$ be the Liouville or the Möbius function, and suppose that $f$ admits correlations for logarithmic averages on $\mathbf{I}=\left(\left[N_{k}\right]\right)_{k \in \mathbb{N}}, N_{k} \rightarrow \infty$. If $\|f\|_{U_{*, \text { log }}^{s}(\mathbf{I})}=0$, then $f$ satisfies the logarithmic Chowla conjecture on $\mathbf{I}$ for correlations involving $s+1$ terms.

Remarks. - The equivalence is proved in [42] only when $N_{k}=k, k \in \mathbb{N}$, but the argument in [42] also gives the stated result.

- An extension of this result that covers more general multiplicative functions is suggested in [42, Remarks 1.10 and 3.5]. We give a related result in Theorem 1.8 below.

- The two main ingredients used in the proof of Theorem 1.7 were a newly devised "entropy decrement" argument from [41] and the Gowers uniformity of the $W$-tricked von Mangoldt function established in $[23,24,26]$.

In order to obtain Theorem 1.4 we use the following variant of the previous result which is established in Section 2.7. The starting point of the proof is an identity for general sequences (see Proposition 2.11) which is implicit in [41].

Theorem 1.8. Let $f_{1} \in \mathcal{M}$ be a multiplicative function which admits correlations for logarithmic averages on $\mathbf{I}=\left(\left[N_{k}\right]\right)_{k \in \mathbb{N}}, N_{k} \rightarrow \infty$, and satisfies $\left\|f_{1}\right\|_{U_{\text {log }}^{s}(\mathbf{I})}=0$ for some $s \geq 2$. Then

$$
\mathbb{E}_{m \in \mathbf{I}}^{\log } f_{1}\left(m+n_{1}\right) \cdots f_{s}\left(m+n_{s}\right)=0
$$

holds for all $f_{2}, \ldots, f_{s} \in \mathcal{M}$ and distinct $n_{1}, \ldots, n_{s} \in \mathbb{N}$. 


\section{NIKOS FRANTZIKINAKIS}

\subsection{A problem}

The previous results motivate the following problem:

Problem. Let $f \in \mathcal{M}$ be a strongly aperiodic multiplicative function which admits correlations for Cesàro (or logarithmic) averages on $\mathbf{I}=\left(\left[N_{k}\right]\right)_{k \in \mathbb{N}}, N_{k} \rightarrow \infty$. Show that the sequence $(f(n))_{n \in \mathbb{N}}$ is ergodic on $\mathbf{I}$ for Cesàro (corr. logarithmic) averages.

Remark. In fact, it seems likely that every real valued bounded multiplicative function is ergodic for Cesàro averages on $\mathbf{I}=([N])_{N \in \mathbb{N}}$.

A solution to this problem for logarithmic averages for the Liouville (or the Möbius) function, coupled with Corollary 1.2, would imply that the Liouville (or the Möbius) function satisfies the Chowla conjecture, and hence the Sarnak conjecture, for logarithmic averages. It would also imply that all possible sign patterns are taken by consecutive values of $\lambda$, and that each size $\ell$ pattern with logarithmic density $2^{-\ell}$, and as a consequence, with upper natural density greater than $2^{-\ell}$.

Currently, we cannot even exclude the (unlikely) possibility that $\lambda$ is generic for a measure on $\{-1,1\}^{\mathbb{N}}$ which induces a measure preserving system with ergodic components circle rotations.

\section{Acknowledgement}

I would like to thank B. Host for various useful discussions during the preparation of this article and B. Kra and P. Sarnak for useful remarks.

\section{Background, notation, and tools}

In this section we define some concepts used throughout the article.

\subsection{Cesàro and logarithmic averages}

Recall that for $N \in \mathbb{N}$ we let $[N]=\{1, \ldots, N\}$. If $A$ is a finite non-empty subset of $\mathbb{N}$ and $a: A \rightarrow \mathbb{C}$, then we define the

- Cesàro average of $(a(n))_{n \in A}$ on $A$ to be

$$
\mathbb{E}_{n \in A} a(n):=\frac{1}{|A|} \sum_{n \in A} a(n)
$$

- logarithmic average of $(a(n))_{n \in A}$ on $A$ to be

$$
\mathbb{E}_{n \in A}^{\log } a(n):=\frac{1}{\sum_{n \in A} \frac{1}{n}} \sum_{n \in A} \frac{a(n)}{n} .
$$

We say that the sequence of intervals $\mathbf{I}=\left(I_{N}\right)_{N \in \mathbb{N}}$ is a Folner sequence for 
- Cesàro averages if $\lim _{N \rightarrow \infty}\left|I_{N}\right|=\infty$;

- logarithmic averages if $\lim _{N \rightarrow \infty} \sum_{n \in I_{N}} \frac{1}{n}=\infty$.

If $I_{N}=\left(a_{N}, b_{N}\right), N \in \mathbb{N}$, the second condition is equivalent to $b_{N} / a_{N} \rightarrow \infty$.

If $a: \mathbb{N} \rightarrow \mathbb{C}$ is a bounded sequence, and $\mathbf{I}=\left(I_{N}\right)_{N \in \mathbb{N}}$ is a Følner sequence of intervals for Cesàro or logarithmic averages, we define the

- Cesàro mean of $(a(n))_{n \in \mathbb{N}}$ on $\mathbf{I}$ to be

$$
\mathbb{E}_{n \in \mathbf{I}} a(n):=\lim _{N \rightarrow \infty} \mathbb{E}_{n \in I_{N}} a(n)
$$

if the limit exists;

- logarithmic mean of $(a(n))_{n \in \mathbb{N}}$ on $\mathbf{I}$ to be

$$
\mathbb{E}_{n \in \mathbf{I}}^{\log } a(n):=\lim _{N \rightarrow \infty} \mathbb{E}_{n \in I_{N}}^{\log } a(n)
$$

if the limit exists.

- If the previous mean values exist for every Følner sequence of intervals $\mathbf{I}$, then we denote the common mean value by $\mathbb{E}_{n \in \mathbb{N}} a(n)$ and $\mathbb{E}_{n \in \mathbb{N}}^{\log } a(n)$ respectively.

Note that all these mean values are shift invariant, meaning, for every $a \in \ell^{\infty}(\mathbb{N})$ and $h \in \mathbb{N}$ the sequences $(a(n))_{n \in \mathbb{N}}$ and $(a(n+h))_{n \in \mathbb{N}}$ have the same Cesàro/logarithmic mean on $\mathbf{I}$.

It is easy to see using partial summation, that if $(a(n))_{n \in \mathbb{N}}$ has a mean value on $\mathbf{I}=([N])_{N \in \mathbb{N}}$, then its logarithmic mean on $\mathbf{I}=([N])_{N \in \mathbb{N}}$ exists and the two means coincide. Note though that a similar statement fails for some subsequences $\mathbf{I}=\left(\left[N_{k}\right]\right)_{k \in \mathbb{N}}$ with $N_{k} \rightarrow \infty$, and also the converse implication fails for $\mathbf{I}=([N])_{N \in \mathbb{N}}$.

Definition 2.1. Let $\mathbf{I}=\left(I_{N}\right)_{N \in \mathbb{N}}$ be a sequence of intervals with $\left|I_{N}\right| \rightarrow \infty$. We say that the sequence $a \in \ell^{\infty}(\mathbb{N})$ satisfies the Chowla conjecture for Cesàro averages on $\mathbf{I}$ if

$$
\mathbb{E}_{m \in \mathbf{I}} a\left(c_{1} m+n_{1}\right) \cdots a\left(c_{s} m+n_{s}\right)=0,
$$

for all $s \in \mathbb{N}, c_{1}, \ldots, c_{s} \in \mathbb{N}$, and non-negative integers $n_{1}, \ldots, n_{s}$ such that $c_{i} n_{j} \neq c_{j} n_{i}$ for all $i \neq j$.

Similar definitions apply for logarithmic averages and when we restrict the number of terms in the product.

\subsection{Measure preserving systems}

A measure preserving system, or simply a system, is a quadruple $(X, X, \mu, T)$ where $(X, X, \mu)$ is a probability space and $T: X \rightarrow X$ is an invertible measure preserving transformation. The system is ergodic if the only sets that are invariant by $T$ have measure 0 or 1 . The von Neumann ergodic theorem implies that for ergodic systems we have

$$
\lim _{N \rightarrow \infty} \mathbb{E}_{n \in I_{N}} \int T^{n} F \cdot G d \mu=\int F d \mu \int G d \mu,
$$

for every sequence of intervals $\left(I_{N}\right)_{N \in \mathbb{N}}$ with $\left|I_{N}\right| \rightarrow \infty$ and functions $F, G \in L^{2}(\mu)$. In the previous statement and throughout, with $T F$ we denote the composition $F \circ T$ and with $T^{n}$ we denote the composition $T \circ \cdots \circ T$. 


\section{NIKOS FRANTZIKINAKIS}

\subsection{Ergodicity of sequences}

To each bounded sequence that is distributed "regularly" along a sequence of intervals with lengths increasing to infinity, we associate a measure preserving system; the notion of ergodicity of this sequence is then naturally inherited from the corresponding property of the system.

Definition 2.2. Let $\mathbf{I}:=\left(I_{N}\right)_{N \in \mathbb{N}}$ be a sequence of intervals with $\left|I_{N}\right| \rightarrow \infty$. We say that the sequence $a \in \ell^{\infty}(\mathbb{N})$ admits correlations for Cesàro averages on $\mathbf{I}$, if the limit

$$
\lim _{N \rightarrow \infty} \mathbb{E}_{m \in I_{N}} b_{1}\left(m+n_{1}\right) \cdots b_{s}\left(m+n_{s}\right)
$$

exists, for every $s \in \mathbb{N}, n_{1}, \ldots, n_{s} \in \mathbb{N}$ (not necessarily distinct), and all sequences $b_{1}, \ldots, b_{s}$ that belong to the set $\{a, \bar{a}\}$.

A similar definition applies for logarithmic averages; in place of $\mathbb{E}_{m \in I_{N}}$ use $\mathbb{E}_{m \in I_{N}}^{\log }$.

Remark. If $a \in \ell^{\infty}(\mathbb{Z})$, then using a diagonal argument we get that any sequence of intervals $\mathbf{I}=\left(I_{N}\right)_{N \in \mathbb{N}}$ has a subsequence $\mathbf{I}^{\prime}=\left(I_{N_{k}}\right)_{k \in \mathbb{N}}$, such that the sequence $(a(n))_{n \in \mathbb{N}}$ admits correlations on $\mathbf{I}^{\prime}$.

The correspondence principle of Furstenberg was originally used in [17] in order to translate Szemerédi's theorem on arithmetic progressions to an ergodic statement. We will use the following variant which applies to general bounded sequences:

Proposition 2.3. Let $a \in \ell^{\infty}(\mathbb{N})$ be a sequence that admits correlations for Cesàro averages on the sequence of intervals $\mathbf{I}:=\left(I_{N}\right)_{N \in \mathbb{N}}$ with $\left|I_{N}\right| \rightarrow \infty$. Then there exist a system $(X, X, \mu, T)$ and a function $F \in L^{\infty}(\mu)$, such that

$$
\mathbb{E}_{m \in \mathbf{I}} a_{1}\left(m+n_{1}\right) \cdots a_{s}\left(m+n_{s}\right)=\int T^{n_{1}} F_{1} \cdots T^{n_{s}} F_{s} d \mu,
$$

for every $s \in \mathbb{N}, n_{1}, \ldots, n_{s} \in \mathbb{N}$, where for $j=1, \ldots, s$ the sequence $a_{j}$ is either $a$ or $\bar{a}$ and $F_{j}$ is $F$ or $\bar{F}$ respectively. A similar statement holds for logarithmic averages.

Remark. For sequences bounded by 1 , in the previous correspondence, $X, X, T$, and $F$ can be taken to be independent of the sequence $a$ and $\mathbf{I}$, and it is only the measure $\mu$ that varies. Furthermore, the system constructed is uniquely determined up to isomorphism by the pair $(a, \mathbf{I})$.

Proof. Let $X:=D^{\mathbb{Z}}$, where $D$ is the closed disk in $\mathbb{C}$ of radius $\|a\|_{\infty}$, be endowed with the product topology and with the invertible and continuous shift $T$ given by $(T x)(k)=x(k+1), k \in \mathbb{Z}$. We define $F \in C(X)$ by $F(x):=x(0), x \in X$, and $\omega \in D^{\mathbb{Z}}$ by $\omega(k):=a(k)$ for $k \in \mathbb{N}$ and $\omega(k)=0$ for $k \leq 0$. Lastly, we let $\mu$ be a $w^{*}$-limit point for the sequence of measures $\mu_{N}:=\frac{1}{\mid I_{N}} \sum_{n \in I_{N}} \delta_{T^{n} \omega}, N \in \mathbb{N}$. Then $\mu$ is a $T$-invariant probability measure on $X$, and since $F\left(T^{n} \omega\right)=a(n)$ for $n \in \mathbb{N}$ and $(a(n))_{n \in \mathbb{N}}$ admits correlations for Cesàro averages on $\mathbf{I}$, the asserted identity follows immediately.

Definition 2.4. Let $a \in \ell^{\infty}(\mathbb{N})$ be a sequence that admits correlations for Cesàro averages on the sequence of intervals $\mathbf{I}:=\left(I_{N}\right)_{N \in \mathbb{N}}$ with $\left|I_{N}\right| \rightarrow \infty$. We call the system defined in Proposition 2.3 the Furstenberg system induced by a and $\mathbf{I}$ for Cesàro averages.

A similar definition applies for logarithmic averages. 
Remarks. - A priori a sequence $a \in \ell^{\infty}(\mathbb{Z})$ may have uncountably many non-isomorphic Furstenberg systems depending on which sequence of intervals I we choose to work with. Furthermore, for fixed $(a, \mathbf{I})$ the Furstenberg systems associated with Cesáro and logarithmic averages could be very different.

- If we assume that the Liouville function admits correlations on $([N])_{N \in \mathbb{N}}$, then the corresponding Furstenberg system is the Liouville system alluded to in the introduction.

Definition 2.5. Let $\mathbf{I}=\left(I_{N}\right)_{N \in \mathbb{N}}$ be a sequence of intervals with $\left|I_{N}\right| \rightarrow \infty$. We say that a sequence $a \in \ell^{\infty}(\mathbb{N})$ is ergodic for Cesàro averages on $\mathbf{I}$ if

1. it admits correlations for Cesàro averages on $\mathbf{I}$; and

2. the induced measure preserving system for Cesàro averages is ergodic.

A similar definition applies for logarithmic averages in which case we say that $a \in \ell^{\infty}(\mathbb{N})$ is ergodic for logarithmic averages on $\mathbf{I}$.

Note that the second condition for Cesàro averages is equivalent to having the identities

$$
\lim _{N \rightarrow \infty} \mathbb{E}_{n \in[N]}\left(\mathbb{E}_{m \in \mathbf{I}} b(m+n) \cdot c(m)\right)=\mathbb{E}_{m \in \mathbf{I}} b(m) \cdot \mathbb{E}_{m \in \mathbf{I}} c(m),
$$

for all $b, c \in \ell^{\infty}(\mathbb{N})$ of the form $b(m)=a_{1}\left(m+h_{1}\right) \cdots a_{s}\left(m+h_{s}\right), m \in \mathbb{N}$, for some $s \in \mathbb{N}$, non-negative integers $h_{1}, \ldots, h_{s}$, and $a_{i} \in\{a, \bar{a}\}$, and similarly for $(c(m))_{m \in \mathbb{N}}$. For logarithmic averages a similar condition holds with $\mathbb{E}_{m \in \mathbf{I}}$ replaced by $\mathbb{E}_{m \in \mathbf{I}}^{\log }$.

\subsection{Ergodic seminorms and the factors $z_{s}$.}

Following [28], if $(X, X, \mu, T)$ is a system we define the Host-Kra seminorms of $F \in L^{\infty}(\mu)$ inductively by

$$
\|F\|_{1}^{2}:=\mathbb{E}_{h \in \mathbb{N}} \int T^{h} F \cdot \bar{F} d \mu, \quad\|F\|_{s+1}^{2^{s+1}}:=\mathbb{E}_{h \in \mathbb{N}}\left\|T^{h} F \cdot \bar{F}\right\|_{s}^{2^{s}},
$$

for $s \in \mathbb{N}$, where the implicit limits defining the mean values $\mathbb{E}_{h \in \mathbb{N}}$ are known to exist by [28]. It is also shown in the same article that for every $s \in \mathbb{N}$ there exist $T$-invariant sub- $\sigma$-algebras $z_{s}$ of $L^{\infty}(\mu)$ such that $\|F\|_{s+1}=0$ if and only if $F \perp L^{2}\left(z_{s}\right)$, meaning $\int F \cdot G d \mu=0$ for every $G \in L^{2}(\mu)$ that is $z_{s}$-measurable. We remark that the previous properties are proved in [28] only for ergodic systems but similar arguments apply for general systems. We are going to use the following important structure theorem (nilsystems are defined in Section 3.1):

Theorem 2.6 (Host, Kra [28]). Let $(X, X, \mu, T)$ be an ergodic system and $s \in \mathbb{N}$. Then the system $\left(X, Z_{s}, \mu, T\right)$ is an inverse limit of s-step nilsystems.

The last property means that there exist $T$-invariant sub- $\sigma$-algebras $z_{s, n}, n \in \mathbb{N}$, that span $z_{s}$, such that for every $n \in \mathbb{N}$ the factor system associated with $z_{s, n}$ is isomorphic to an $s$-step nilsystem. 


\section{NIKOS FRANTZIKINAKIS}

\subsection{Local uniformity seminorms}

Let $\mathbf{I}=\left(I_{N}\right)_{N \in \mathbb{N}}$ be a sequence of intervals with $\left|I_{N}\right| \rightarrow \infty$ and $a \in \ell^{\infty}(\mathbb{N})$ be a sequence that admits correlations for Cesàro averages on I. Following [29], we define the uniformity seminorms $\|a\|_{U^{s}(\mathbf{I})}$ inductively as follows:

$$
\|a\|_{U^{1}(\mathbf{I})}^{2}:=\mathbb{E}_{h \in \mathbb{N}}\left(\mathbb{E}_{n \in \mathbf{I}} a(n+h) \overline{a(n)}\right)
$$

(by van der Corput's lemma $\left|\mathbb{E}_{n \in \mathbf{I}} a(n)\right| \leq 4\|a\|_{U^{1}(\mathbf{I})}$ ) and for $s \in \mathbb{N}$

$$
\|a\|_{U^{s+1}(\mathbf{I})}^{s^{s+1}}:=\mathbb{E}_{h \in \mathbb{N}}\left\|S_{h} a \cdot \bar{a}\right\|_{U^{s}(\mathbf{I})}^{2^{s}},
$$

where $S_{h}$ is the shift operator on $\ell^{\infty}(\mathbb{N})$ defined for $h \in \mathbb{N}$ by

$$
\left(S_{h} a\right)(n):=a(n+h), \quad n \in \mathbb{N} .
$$

It is not immediately clear that all the iterative limits defining the above averages exist. This can be proved by reinterpreting these seminorms in ergodic terms using the measure preserving system $(X, X, \mu, T)$ and the function $F \in L^{\infty}(\mu)$ induced by $a$ and $\mathbf{I}$; we then have $\|a\|_{U^{s}(\mathbf{I})}=\|F\|_{s}$ where $\|F\|_{s}$ is defined as in Section 2.4. Using the ergodic reinterpretation and [28, Theorem 1.2] we deduce the identity

$$
\|a\|_{U^{s}(\mathbf{I})}^{2^{s}}=\mathbb{E}_{\underline{h} \in \mathbb{N}^{s}}\left(\mathbb{E}_{n \in \mathbf{I}} \prod_{\varepsilon \in[s]} \mathcal{C}^{|\varepsilon|} a(n+\varepsilon \cdot \underline{h})\right),
$$

where

$$
\mathcal{C}^{k}(z):= \begin{cases}z & \text { if } k \text { is even } \\ \bar{z} & \text { if } k \text { is odd }\end{cases}
$$

and for $s \in \mathbb{N}$ we let $\underline{0}:=(0, \ldots, 0)$,

$$
\left[[s]:=\{0,1\}^{s}, \quad\left[[s]^{*}:=[[s] \rrbracket \backslash\{\underline{0}\},\right.\right.
$$

and for $\varepsilon=\left(\varepsilon_{1}, \ldots, \varepsilon_{s}\right)$ we let

$$
|\varepsilon|:=\varepsilon_{1}+\cdots+\varepsilon_{s}
$$

Furthermore, the limit $\mathbb{E}_{\underline{h} \in \mathbb{N}^{s}}$ can be defined using averages taken over arbitrary Følner sequences of subsets of $\mathbb{N}^{s}$, or can be defined using the iterative limit $\mathbb{E}_{h_{s} \in \mathbb{N}} \cdots \mathbb{E}_{h_{1} \in \mathbb{N}}$. All these limits exist and are equal; this follows from [28, Theorem 1.2]. It is shown in [28] that $\|F\|_{s} \leq\|F\|_{s+1}$ for every $F \in L^{\infty}(\mu)$ and $s \in \mathbb{N}$; we deduce that

$$
\|a\|_{U^{s}(\mathbf{I})} \leq\|a\|_{U^{s+1}(\mathbf{I})}, \quad \text { for every } s \in \mathbb{N} .
$$

In a similar fashion, if $a \in \ell^{\infty}(\mathbb{N})$ admits correlations for logarithmic averages on $\mathbf{I}$, we define the uniformity seminorms for logarithmic averages $\|a\|_{U_{\log }^{s}(\mathbf{I})}$ as follows:

$$
\|a\|_{U_{\log }^{1}(\mathbf{I})}^{2}:=\mathbb{E}_{h \in \mathbb{N}}\left(\mathbb{E}_{n \in \mathbf{I}}^{\log } a(n+h) \overline{a(n)}\right)
$$

and for $s \in \mathbb{N}$

$$
\|a\|_{U_{\log }^{s+1}(\mathbf{I})}^{2^{s+1}}:=\mathbb{E}_{h \in \mathbb{N}}\left\|S_{h} a \cdot \bar{a}\right\|_{U_{\log }^{s}(\mathbf{I})}^{2^{s}} .
$$




\section{ERgodicity of the Liouville System Implies the Chowla Conjecture}

All implicit limits defining the mean values $\mathbb{E}_{h \in \mathbb{N}}$ can be shown to exist. Note that in the definition of the uniformity seminorms for logarithmic averages only the inner-most average is logarithmic, the others can be given by any shift invariant averaging scheme we like. For example, we have

$$
\|a\|_{U_{\log }^{2}(\mathbf{I})}^{4}=\mathbb{E}_{\left(h_{1}, h_{2}\right) \in \mathbb{N}^{2}}\left(\mathbb{E}_{n \in \mathbf{I}}^{\log } a\left(n+h_{1}+h_{2}\right) \cdot \overline{a\left(n+h_{1}\right)} \cdot \overline{a\left(n+h_{2}\right)} \cdot a(n)\right) .
$$

We also use variants of some local uniformity seminorms introduced by Tao in [42] when $\mathbf{I}=([N])_{N \in \mathbb{N}}$. For $s \in \mathbb{N}, a \in \ell^{\infty}(\mathbb{N})$, and Følner sequence of intervals $\mathbf{I}$, we let

$$
\|a\|_{U_{*}^{s}(\mathbf{I})}:=\limsup _{H \rightarrow \infty} \limsup _{N \rightarrow \infty} \mathbb{E}_{n \in I_{N}}\left\|S_{n} a\right\|_{U^{s}[H]}
$$

(where $\left.\left(S_{n} a\right)(m):=a(n+m)\right)$ and

$$
\|a\|_{U_{*, \log }^{s}(\mathbf{I})}:=\limsup _{H \rightarrow \infty} \limsup _{N \rightarrow \infty} \mathbb{E}_{n \in I_{N}}^{\log }\left\|S_{n} a\right\|_{U^{s}[H]} .
$$

We used that

$$
\|a\|_{U^{s}[N]}:=\frac{\left\|a \cdot \mathbf{1}_{[N]}\right\|_{U^{s}\left(\mathbb{Z}_{2 N}\right)}}{\left\|\mathbf{1}_{[N]}\right\|_{U^{s}\left(\mathbb{Z}_{2 N}\right)}},
$$

([14, Lemma A.2] explains why $2 N$ is an appropriate choice) where $\mathbb{Z}_{N}:=\mathbb{Z} /(N \mathbb{Z})$ and $\|a\|_{U^{s}\left(\mathbb{Z}_{N}\right)}$ are the Gowers uniformity norms. These were defined in [20] as follows:

$$
\|a\|_{U^{1}\left(\mathbb{Z}_{N}\right)}:=\left|\mathbb{E}_{m \in \mathbb{Z}_{N}} a(m)\right|
$$

and for $s \in \mathbb{N}$

$$
\|a\|_{U^{s+1}\left(\mathbb{Z}_{N}\right)}^{2^{s+1}}:=\mathbb{E}_{h \in \mathbb{Z}_{N}}\left\|S_{h} a \cdot \bar{a}\right\|_{U^{s}\left(\mathbb{Z}_{N}\right)}^{2^{s}},
$$

where for $N \in \mathbb{N}$ we use the periodic extension of $a \cdot \mathbf{1}_{[N]}$ to $\mathbb{Z}_{N}$ in the previous computations, or equivalently, we define $\left(S_{h} a\right)(n):=a(n+h \bmod N)$ for $n \in \mathbb{Z}_{N}$.

Proposition 2.7. Let $s \in \mathbb{N}$. If $a \in \ell^{\infty}(\mathbb{N})$ is a sequence that admits correlations for Cesàro averages on the sequence of intervals $\mathbf{I}$, then

$$
\|a\|_{U_{*}^{s}(\mathbf{I})} \leq 4\|a\|_{U^{s}(\mathbf{I})} .
$$

A similar statement holds for logarithmic averages on $\mathbf{I}$ and the corresponding estimate is $\|a\|_{U_{*, \text { log }}^{s}(\mathbf{I})} \leq$ $4\|a\|_{U_{\text {log }}^{s}(\mathbf{I})}$.

Proof. Let $H \in \mathbb{N}$ and $a: \mathbb{Z}_{H} \rightarrow \mathbb{C}$ be bounded by 1 . Then arguing as in the proof of Proposition 3.2 in [6], we get that for every $H_{1}, \ldots, H_{s} \in \mathbb{N}$ the following estimate holds

$$
\|a\|_{U^{s}\left(\mathbb{Z}_{H}\right)}^{2^{s}} \leq 2^{s} \mathbb{E}_{h_{1} \in\left[H_{1}\right]} \cdots \mathbb{E}_{h_{s} \in\left[H_{s}\right]}\left(\mathbb{E}_{h \in[H]} \prod_{\varepsilon \in \llbracket s \rrbracket} \mathcal{C}^{|\varepsilon|} a(h+\varepsilon \cdot \underline{h})\right)+\sum_{j=1}^{s} H_{i}^{-1}
$$

Now let $a: \mathbb{N} \rightarrow \mathbb{C}$ be bounded by 1 . For $H \in \mathbb{N}$ we define $a_{H}: \mathbb{Z}_{2 H} \rightarrow \mathbb{C}$ by $a_{H}(n):=a(n) \cdot \mathbf{1}_{[H]}(n)$ for $n \in[2 H]$ and we extend it periodically to $\mathbb{Z}_{2 H}$. Using the definition of $\|a\|_{U^{s}[H]}$ in conjunction with the 


\section{NIKOS FRANTZIKINAKIS}

estimate (5), applied to $a_{H}$, and using that $\left\|\mathbf{1}_{[H]}\right\|_{U^{s}\left(\mathbb{Z}_{2 H}\right)} \geq\left\|\mathbf{1}_{[H]}\right\|_{U^{1}\left(\mathbb{Z}_{2 H}\right)}=\frac{1}{2}$, we deduce that for every $H, H_{1}, \ldots, H_{s} \in \mathbb{N}$ we have

$$
2^{-2^{s}}\|a\|_{U^{s}[H]}^{2^{s}} \leq 2^{s} \mathbb{E}_{h_{1} \in\left[H_{1}\right]} \cdots \mathbb{E}_{h_{s} \in\left[H_{s}\right]}\left(\mathbb{E}_{h \in[2 H]} \prod_{\varepsilon \in \llbracket s \rrbracket} \mathrm{e}^{|\varepsilon|} a_{H}(h+\varepsilon \cdot \underline{h})\right)+\sum_{j=1}^{s} H_{i}^{-1},
$$

where $\underline{h}=\left(h_{1}, \ldots, h_{s}\right)$. Since for $h \in[2 H]$ and $h_{1} \in\left[H_{1}\right], \ldots, h_{s} \in\left[H_{s}\right]$ we have $a_{H}(h+\varepsilon \cdot \underline{h})=(a$. $\left.\mathbf{1}_{[H]}\right)(h+\varepsilon \cdot \underline{h})$ except possibly when $h \in\left[H-\left(H_{1}+\cdots+H_{s}\right), H\right] \cup\left[2 H-\left(H_{1}+\cdots+H_{s}\right), 2 H\right]$, we get

$$
2^{-2^{s}}\|a\|_{U^{s}[H]}^{2^{s}} \leq 2^{s} \mathbb{E}_{h_{1} \in\left[H_{1}\right]} \cdots \mathbb{E}_{h_{s} \in\left[H_{s}\right]}\left(\mathbb{E}_{h \in[H]} \prod_{\varepsilon \in \llbracket s]} \mathcal{C}^{|\varepsilon|} a(h+\varepsilon \cdot \underline{h})\right)+\sum_{j=1}^{s} H_{i}^{-1}+2^{s} H^{-1} \sum_{j=1}^{s} H_{j},
$$

where the sums $h+\varepsilon \cdot \underline{h}$ are taken in $\mathbb{N}$. Using this estimate for the sequence $S_{n} a$, averaging over $n \in I_{N}$, taking $N \rightarrow \infty$, and then making the change of variables $n \mapsto n-h$, we get that for every $H, H_{1}, \ldots, H_{s} \in \mathbb{N}$ we have

$$
\begin{aligned}
& 2^{-2^{s}} \limsup _{N \rightarrow \infty} \mathbb{E}_{n \in I_{N}}\left\|S_{n} a\right\|_{U^{s}[H]}^{2^{s}} \leq \\
& \qquad 2^{s} \mathbb{E}_{h_{1} \in\left[H_{1}\right]} \cdots \mathbb{E}_{h_{s} \in\left[H_{s}\right]}\left(\mathbb{E}_{n \in \mathbf{I}} \prod_{\varepsilon \in \llbracket s \rrbracket} \mathcal{C}^{|\varepsilon|} a(n+\varepsilon \cdot \underline{h})\right)+\sum_{j=1}^{s} H_{i}^{-1}+2^{s} H^{-1} \sum_{j=1}^{s} H_{j} .
\end{aligned}
$$

Finally, recall that $\|a\|_{U_{*}^{s}(\mathbf{I})}=\lim \sup _{H \rightarrow \infty} \lim \sup _{N \rightarrow \infty} \mathbb{E}_{n \in I_{N}}\left\|S_{n} a\right\|_{U^{s}\left(\mathbb{Z}_{H}\right)}$. Thus, if on the last estimate we take $H \rightarrow \infty$ and then let $H_{S} \rightarrow \infty, \ldots, H_{1} \rightarrow \infty$, we get that

$$
2^{-2^{s}}\|a\|_{U_{*}^{s}(\mathbf{I})}^{2^{s}} \leq 2^{s} \mathbb{E}_{h_{1} \in \mathbb{N}} \cdots \mathbb{E}_{h_{s} \in \mathbb{N}}\left(\mathbb{E}_{n \in \mathbf{I}} \prod_{\varepsilon \in \llbracket s \rrbracket} \mathcal{C}^{|\varepsilon|} a(n+\mathcal{\varepsilon} \cdot \underline{h})\right)=2^{s}\|a\|_{U^{s}(\mathbf{I})}^{2^{s}} .
$$

This proves the first estimate. The proof of the second estimate is similar.

\subsection{Multiplicative functions and strong aperiodicity}

A function $f: \mathbb{N} \rightarrow \mathbb{C}$ is called multiplicative if

$$
f(m n)=f(m) f(n) \text { whenever }(m, n)=1 .
$$

It is called completely multiplicative if the previous identity holds for every $m, n \in \mathbb{N}$. We let

$$
\mathcal{M}:=\{f: \mathbb{N} \rightarrow \mathbb{C} \text { is multiplicative, bounded, and }|f(p)|=1 \text { for every } p \in \mathbb{P}\} .
$$

A Dirichlet character is a periodic completely multiplicative function $\chi$ with $\chi(1)=1$. We say that $f \in \mathcal{M}$ is aperiodic (or non-pretentious following [21]) if

$$
\lim _{N \rightarrow \infty} \mathbb{E}_{n \in[N]} f(a n+b)=0, \quad \text { for all } a, b \in \mathbb{N},
$$

or equivalently, if $\lim _{N \rightarrow \infty} \mathbb{E}_{n \in[N]} f(n) \chi(n)=0$ for every Dirichlet character $\chi$.

The uniformity result stated in Theorem 1.6 holds for a class of multiplicative functions that satisfy a condition introduced in [35] which is somewhat stronger than aperiodicity. In order to state it we need the notion of the distance between two multiplicative functions defined as in [21]: 
Definition 2.8. Let $\mathbb{P}$ be the set of primes. We let $\mathbb{D}: \mathcal{M} \times \mathcal{M} \rightarrow[0, \infty]$ be given by

$$
\mathbb{D}(f, g)^{2}=\sum_{p \in \mathbb{P}} \frac{1}{p}(1-\Re(f(p) \overline{g(p)}))
$$

where $\mathfrak{R}(z)$ denotes the real part of a compelx number $z$. We also let $\mathbb{D}: \mathcal{M} \times \mathcal{M} \times \mathbb{N} \rightarrow[0, \infty]$ be given by

$$
\mathbb{D}(f, g ; N)^{2}=\sum_{p \in \mathbb{P} \cap[N]} \frac{1}{p}(1-\Re(f(p) \overline{g(p)}))
$$

and $M: \mathcal{M} \times \mathbb{N} \rightarrow[0, \infty)$ be given by

$$
M(f ; N):=\min _{|t| \leq N} \mathbb{D}\left(f, n^{i t} ; N\right)^{2} .
$$

A celebrated theorem of Halász [27] states that a multiplicative function $f \in \mathcal{M}$ has zero mean value if and only if for every $t \in \mathbb{R}$ we either have $\mathbb{D}\left(f, n^{i t}\right)=\infty$ or $f\left(2^{k}\right)=-2^{i k t}$ for all $k \in \mathbb{N}$. For our purposes we need information on averages of multiplicative functions taken on typical short intervals. Such results were obtained in $[34,35]$, under conditions that motivate the following definition:

Definition 2.9. The multiplicative function $f \in \mathcal{M}$ is strongly aperiodic if $M(f \cdot \chi ; N) \rightarrow \infty$ as $N \rightarrow \infty$ for every Dirichlet character $\chi$.

Note that strong aperiodicity implies aperiodicity. The converse is not in general true (see [35, Theorem B.1]), but it is true for (bounded) real valued multiplicative functions (see [35, Appendix C]). In particular, the Liouville and the Möbius function are strongly aperiodic. Furthermore, if $f \in \mathcal{M}$ satisfies

1. $f(p)$ is a $d$-th root of unity for all but finitely many primes $p$; and

2. $\mathbb{D}(f, \chi)=\infty$ for every Dirichlet character $\chi$,

then $f$ is strongly aperiodic (see [13, Proposition 6.1]).

We will need the following result; a quantitative variant of which is implicit in [35] (the stated version is also deduced from [35, Theorem A.1] in [13, Theorem 4.1]):

Proposition 2.10 (Matomäki, Radziwiłł, Tao [35]). Let $f \in \mathcal{M}$ be a strongly aperiodic multiplicative function that admits correlations for Cesàro averages on the sequence of intervals $\mathbf{I}:=\left(\left[N_{k}\right]\right)_{k \in \mathbb{N}}$ with $N_{k} \rightarrow \infty$. Then

$$
\lim _{H \rightarrow \infty} \mathbb{E}_{h \in[H]}\left|\mathbb{E}_{n \in \mathbf{I}} f(n+h) \cdot \overline{f(n)}\right|=0 .
$$

A similar statement also holds for logarithmic averages on $\mathbf{I}$.

Remark. It follows from [35, Theorem B.1] that strong aperiodicity cannot be replaced by aperiodicity; in particular, there exist an aperiodic multiplicative function $f \in \mathcal{M}$, a positive constant $c$, and a sequence of intervals $\mathbf{I}:=\left(\left[N_{k}\right]\right)_{k \in \mathbb{N}}$ with $N_{k} \rightarrow \infty$, such that

$$
\left|\mathbb{E}_{n \in \mathbf{I}} f(n+h) \cdot \overline{f(n)}\right| \geq c, \quad \text { for every } h \in \mathbb{N} .
$$




\section{NIKOS FRANTZIKINAKIS}

\subsection{Local uniformity implies the Elliott conjecture}

In this subsection we prove Theorem 1.8 by adapting the argument in [41] which deals with the case where all the multiplicative functions are equal to the Liouville or the Möbius function. In what follows, if $(a(p))_{p \in \mathbb{P}}$ is a sequence indexed by the primes, we denote by $\mathbb{E}_{p \in \mathbb{P}} a(p)$ the $\operatorname{limit} \lim _{N \rightarrow \infty} \frac{\log N}{N} \sum_{p \leq N} a(p)$ if it exists.

Our starting point is the following identity which is implicit in [41] and its proof was sketched in [15, Appendix C] (see also [43, Theorem 3.6] for a variant of this identity):

Proposition 2.11. Let $\mathbf{I}=\left(\left[N_{k}\right]\right)_{k \in \mathbb{N}}$ be a sequence of intervals with $N_{k} \rightarrow \infty,\left(c_{p}\right)_{p \in \mathbb{P}}$ be a bounded sequence of complex numbers, $s \in \mathbb{N}, a_{1}, \ldots, a_{s} \in \ell^{\infty}(\mathbb{N})$, and $n_{1}, \ldots, n_{s} \in \mathbb{N}$. Then, assuming that on the left and right hand side below the limit $\mathbb{E}_{m \in \mathbf{I}}^{\log }$ exists for every $p \in \mathbb{P}$ and the limit $\mathbb{E}_{p \in \mathbb{P}}$ exists, we have

$$
\mathbb{E}_{p \in \mathbb{P}} c_{p}\left(\mathbb{E}_{m \in \mathbf{I}}^{\log } \prod_{j=1}^{s} a_{j}\left(p m+p n_{j}\right)\right)=\mathbb{E}_{p \in \mathbb{P}} c_{p}\left(\mathbb{E}_{m \in \mathbf{I}}^{\log } \prod_{j=1}^{s} a_{j}\left(m+p n_{j}\right)\right) .
$$

We deduce from this the following identity for multiplicative functions:

Corollary 2.12. Let $\mathbf{I}=\left(\left[N_{k}\right]\right)_{k \in \mathbb{N}}$ be a sequence of intervals with $N_{k} \rightarrow \infty, s \in \mathbb{N}, f_{1}, \ldots, f_{s} \in \mathcal{M}$, and $n_{1}, \ldots, n_{s} \in \mathbb{N}$. Suppose that for every $p \in \mathbb{P}$ on the left and right hand side below the limit $\mathbb{E}_{m \in \mathbf{I}}^{\log }$ exists and the limit $\mathbb{E}_{p \in \mathbb{P}}$ exists. Furthermore, suppose that the limit $\mathbb{E}_{m \in \mathbf{I}}^{\log } \prod_{j=1}^{s} f_{j}\left(p m+p n_{j}\right)$ exists for every $p \in \mathbb{P}^{2}$ Then we have

$$
\mathbb{E}_{m \in \mathbf{I}}^{\log } \prod_{j=1}^{s} f_{j}\left(m+n_{j}\right)=\mathbb{E}_{p \in \mathbb{P}} c_{p}\left(\mathbb{E}_{m \in \mathbf{I}}^{\log } \prod_{j=1}^{s} f_{j}\left(m+p n_{j}\right)\right) .
$$

where $c_{p}:=\prod_{j=1}^{s} \overline{f_{j}(p)}$ for $p \in \mathbb{P}$.

Proof. For $p \in \mathbb{P}$ and $j=1, \ldots s$, we have $f_{j}\left(p\left(m+n_{j}\right)\right)=f_{j}(p) f_{j}\left(m+n_{j}\right)$ unless $m \equiv-n_{j}(\bmod p)$. Hence,

$$
\mathbb{E}_{m \in \mathbf{I}}^{\log } \prod_{j=1}^{s} f_{j}\left(m+n_{j}\right)=c_{p} \mathbb{E}_{m \in \mathbf{I}}^{\log } \prod_{j=1}^{s} f_{j}\left(p m+p n_{j}\right)+O(1 / p)
$$

where the implicit constant depends only on $s$ and on the sup-norm of $f_{1}, \ldots, f_{s}$. Averaging over $p \in \mathbb{P}$ we get

$$
\mathbb{E}_{m \in \mathbf{I}}^{\log } \prod_{j=1}^{s} f_{j}\left(m+n_{j}\right)=\mathbb{E}_{p \in \mathbb{P}} c_{p}\left(\mathbb{E}_{m \in \mathbf{I}}^{\log } \prod_{j=1}^{s} f_{j}\left(p m+p n_{j}\right)\right) .
$$

Applying Proposition 2.11, we get the asserted identity.

We will also need the following multiple ergodic theorem:

Proposition 2.13. Let $(X, X, \mu, T)$ be a system, $s \geq 2, F_{1}, \ldots, F_{s} \in L^{\infty}(\mu)$, and $n_{1}, \ldots, n_{s} \in \mathbb{N}$ be distinct integers. Suppose that $\left\|F_{1}\right\|_{s}=0$. Then

$$
\mathbb{E}_{p \in \mathbb{P}}\left|\int \prod_{j=1}^{s} T^{p n_{j}} F_{j} d \mu\right|=0 .
$$

\footnotetext{
${ }^{2}$ This assumption can be omitted using a subsequential argument but we will not need this.
} 
Proof. It suffices to show that

$$
\mathbb{E}_{p \in \mathbb{P}} \int \prod_{j=1}^{s}(T \times T)^{p n_{j}}\left(F_{j} \otimes \overline{F_{j}}\right) d(\mu \times \mu)=0 .
$$

For $w \in \mathbb{N}$ let $W$ denote the product of the first $w$ primes. Following the proof of [16, Theorem 1.3] (which uses the Gowers uniformity of the $W$-tricked von Mangoldt function established in $[23,24,26]$ ) we get that the average on the left hand side is equal to

$$
\lim _{W \rightarrow \infty} \mathbb{E}_{(k, W)=1} \mathbb{E}_{n \in \mathbb{N}} \int \prod_{j=1}^{s}(T \times T)^{(n W+k) n_{j}}\left(F_{j} \otimes \overline{F_{j}}\right) d(\mu \times \mu),
$$

where the average $\mathbb{E}_{(k, W)=1}$ is taken over those $k \in\{1, \ldots, W-1\}$ such that $(k, W)=1$. In order to show that this limit vanishes, it suffices to show that for all distinct $l_{1}, \ldots, l_{s} \in \mathbb{N}$ and for arbitrary $k_{1}, \ldots, k_{s} \in \mathbb{N}$ we have

$$
\mathbb{E}_{n \in \mathbb{N}} \int \prod_{j=1}^{s}(T \times T)^{l_{j} n+k_{j}}\left(F_{j} \otimes \overline{F_{j}}\right) d(\mu \times \mu)=0 .
$$

It follows from [31, Theorem A.8] (for $s \geq 3$, but a simple argument works for $s=2$ ) that in order to establish this identity it suffices to show that $\left\|F_{1} \otimes \overline{F_{1}}\right\|_{s-1, T \times T}=0$. But this follows since $\| F_{1} \otimes$ $\overline{F_{1}}\left\|_{s-1, T \times T} \leq\right\| F_{1} \|_{s, T}^{2}$ and by assumption we have $\left\|F_{1}\right\|_{s, T}=0$. This completes the proof.

Proof of Theorem 1.8. Arguing by contradiction, suppose that the conclusion fails. Then there exist multiplicative functions $f_{2}, \ldots, f_{s} \in \mathcal{M}$, distinct $n_{1}, \ldots, n_{s} \in \mathbb{N}$, and a subsequence $\left(N_{k}^{\prime}\right)_{k \in \mathbb{N}}$ of $\left(N_{k}\right)_{k \in \mathbb{N}}$, such that for $\mathbf{I}^{\prime}:=\left(\left[N_{k}^{\prime}\right]\right)_{k \in \mathbb{N}}$ the limit $\mathbb{E}_{m \in \mathbf{I}^{\prime}}^{\log } \prod_{j=1}^{s} g_{j}\left(l_{j} m+k_{j}\right)$ exists for every $s, k_{1}, \ldots, k_{s}, l_{1}, \ldots, l_{s} \in \mathbb{N}$, and $g_{1}, \ldots, g_{s} \in\left\{a_{1}, \ldots, a_{s}, \overline{a_{1}}, \ldots, \overline{a_{s}}\right\}$, and such that

$$
\mathbb{E}_{m \in \mathbf{I}^{\mathbf{I}}}^{\log } \prod_{j=1}^{s} f_{j}\left(m+n_{j}\right) \neq 0 .
$$

Using Corollary 2.12, we will get a contradiction if we show that

$$
\mathbb{E}_{p \in \mathbb{P}}\left|\mathbb{E}_{m \in \mathbf{I}^{\prime}}^{\log } \prod_{j=1}^{s} f_{j}\left(m+p n_{j}\right)\right|=0
$$

In order to prove this identity we will reinterpret it in ergodic terms. Using a variant of Proposition 2.3 which applies to several sequences (see [13, Proposition 3.3]) we get that there exist a system $(X, X, \mu, T)$ and functions $F_{1}, \ldots, F_{s} \in L^{\infty}(\mu)$ such that

$$
\mathbb{E}_{m \in \mathbf{I}^{\prime}}^{\log } \prod_{j=1}^{s} f_{j}\left(m+p n_{j}\right)=\int \prod_{j=1}^{s} T^{p n_{j}} F_{j} d \mu
$$

holds for every $p \in \mathbb{P}$ and

$$
\left\|f_{1}\right\|_{U_{\log }^{s}\left(\mathbf{I}^{\prime}\right)}=\left\|F_{1}\right\|_{s}
$$


Since by assumption $\left\|f_{1}\right\|_{U_{\log }^{s}(\mathbf{I})}=0$ and $\mathbf{I}^{\prime}$ is a subsequence of $\mathbf{I}$, we have $\left\|f_{1}\right\|_{U_{\log }^{s}\left(\mathbf{I}^{\prime}\right)}=0$. Hence, $\left\|F_{1}\right\|_{s}=0$, and Proposition 2.13 gives that

$$
\mathbb{E}_{p \in \mathbb{P}}\left|\int \prod_{j=1}^{s} T^{p n_{j}} F_{j} d \mu\right|=0 .
$$

We deduce from this and identity (7) that equation (6) holds. This completes the proof.

\section{Nilmanifolds, nilcharacters, and nilsequences}

\subsection{Nilmanifolds}

If $G$ is a group we let $G_{1}:=G$ and $G_{j+1}:=\left[G, G_{j}\right], j \in \mathbb{N}$. We say that $G$ is s-step nilpotent if $G_{s+1}$ is the trivial group. An $s$-step nilmanifold is a homogeneous space $X=G / \Gamma$, where $G$ is an $s$-step nilpotent Lie group and $\Gamma$ is a discrete cocompact subgroup of $G$. With $e_{X}$ we denote the image in $X$ of the unit element of $G$. An $s$-step nilsystem is a system of the form $\left(X, G / \Gamma, m_{X}, T_{b}\right)$, where $X=G / \Gamma$ is a $k$-step nilmanifold, $b \in G, T_{b}: X \rightarrow X$ is defined by $T_{b}\left(g \cdot e_{X}\right):=(b g) \cdot e_{X}$ for $g \in G, m_{X}$ is the normalized Haar measure on $X$, and $G / \Gamma$ is the completion of the Borel $\sigma$-algebra of $G / \Gamma$. We call the map $T_{b}$ or the element $b$ a nilrotation. Henceforth, we assume that every nilsystem is equipped with a fixed Riemannian metric $d$. If $\Psi$ is a function on $X$ we let $\|\Psi\|_{\operatorname{Lip}(X)}:=\sup _{x \in X}|\Psi(x)|+\sup _{\substack{x, y \in X \\ x \neq y}} \frac{|\Psi(x)-\Psi(y)|}{d(x, y)}$. With $\operatorname{Lip}(X)$ we denote the set of all functions $\Psi: X \rightarrow \mathbb{C}$ with bounded $\operatorname{Lip}(X)$-norm.

If $H$ is a closed subgroup of $G$, then it is shown in [32, Section 2.2] that the following three properties are equivalent:

- $H \cdot \Gamma$ is closed in $G$;

- $H \cdot e_{X}$ is closed in $X$;

- $H \cap \Gamma$ is cocompact in $H$.

For any such $H$, the nilmanifold $H /(H \cap \Gamma)$ is called a sub-nilmanifold of $X$.

With $G^{0}$ we denote the connected component of the identity element in $G$ (this is a normal subgroup of $G$ ). If the nilsystem is ergodic, then since $G^{\prime}:=\left\langle G^{0}, b\right\rangle$ is a non-empty open subgroup of $G$ that is invariant under $b$, we have $G^{\prime} \cdot e_{X}=X$. Hence, $X=G^{\prime} / \Gamma^{\prime}$ where $\Gamma^{\prime}=G^{\prime} \cap \Gamma$. For every ergodic nilsystem we will use such a representation for $X$ and thus assume that $G=\left\langle G^{0}, b\right\rangle$. This implies (see for example [3, Theorem 4.1]) that for $j \geq 2$ all the commutator subgroups $G_{j}$ are connected.

Throughout the article, in the case of ergodic nilsystems, we are going to use these properties without further reference.

\subsection{Equidistribution}

Let $X=G / \Gamma$ be a nilmanifold. We say that a sequence $g: \mathbb{N} \rightarrow X$ is equidistributed in $X$ if for every $F \in C(X)$ we have

$$
\lim _{N \rightarrow \infty} \mathbb{E}_{n \in[N]} F(g(n))=\int F d m_{X}
$$


where $m_{X}$ denotes the normalized Haar measure on $X$.

It is proved in [33] (see also [32]) that for every $b \in G$ the set $Y=\overline{\left\{b^{n} \cdot e_{X}, n \in \mathbb{N}\right\}}$ is a sub-nilmanifold of $X$, the nilrotation $b$ acts ergodically in $Y$, and the sequence $\left(b^{n} \cdot y\right)_{n \in \mathbb{N}}$ is equidistributed in $Y$ for every $y \in Y$. Furthermore, we can represent $Y$ as $Y=H / \Delta$ where $H$ is a closed subgroup of $G$ that contains the element $b$ (see the remark following [32, Theorem 2.21]). If $Y$ is connected, then for every $k \in \mathbb{N}$ the nilrotation $b^{k}$ acts ergodically on $Y$. If $Y$ is not connected, then there exists $r \in \mathbb{N}$ such that the nilrotation $b^{r}$ acts ergodically on the connected component $Y^{0}$ of $e_{X}$ in $Y$.

\subsection{Vertical nilcharacters on $X$ and on $X^{0}$}

Let $s \in \mathbb{N}$ and $X=G / \Gamma$ be a (not necessarily connected) $s$-step nilmanifold and suppose that $G=\left\langle G^{0}, b\right\rangle$ for some $b \in G$. If $s \geq 2$, then $G_{s}$ is connected and the group $K_{s}:=G_{s} /\left(G_{s} \cap \Gamma\right)$ is a finite dimensional torus (perhaps the trivial one). Let $\widehat{K}_{s}$ be the dual group of $K_{s}$; it consists of the characters of $G_{s}$ that are $\left(\Gamma \cap G_{s}\right)$-invariant. A vertical nilcharacter of $X$ with frequency $\chi$, where $\chi \in \widehat{K}_{s}$, is a function $\Phi \in \operatorname{Lip}(X)$ that satisfies

$$
\Phi(u \cdot x)=\chi(u) \Phi(x) \text {, for every } u \in G_{s} \text { and } x \in X .
$$

If $\chi$ is a non-trivial character of $K_{s}$, we say that $\Phi$ is a non-trivial vertical nilcharacter, otherwise we say that it is a trivial vertical nilcharacter. It is known that the linear span of vertical nilcharacters is dense in $C(X)$ with the uniform norm (see for example [24, Proof of Lemma 2.7] or [41, Excercise 1.6.20]).

If the nilmanifold $X$ is not connected, let $X^{0}$ be the connected component of $e_{X}$ in $X$. We claim that for $s \geq 2$ the restriction of a non-trivial vertical nilcharacter $\Phi$ of $X$ onto $X^{0}$ is a non-trivial vertical nilcharacter of $X^{0}$ with the same frequency. To see this, note first that since $\left(G^{0} \Gamma\right) / \Gamma$ is a non-empty closed and open subset of the connected space $X^{0}$, we have $X^{0}=\left(G^{0} \Gamma\right) / \Gamma$. It thus suffices to show that $\left(G^{0} \Gamma\right)_{s}=G_{s}$. To this end, let $r$ be the smallest integer such that $b^{r} \in G^{0} \Gamma$. Then $G /\left(G^{0} \Gamma\right)$ is isomorphic to the cyclic group $\mathbb{Z}_{r}$. By induction, for every $k \geq 1$ and all $g_{1}, \ldots, g_{k} \in G$, we have

$$
\left[\left[\ldots\left[g_{1}, g_{2}\right], g_{3}\right], \ldots, g_{k}\right]^{r^{k}}=\left[\left[\ldots\left[g_{1}^{r}, g_{2}^{r}\right], g_{3}^{r}\right], \ldots, g_{k}^{r}\right] \bmod G_{k+1}
$$

Letting $k=s$ and using that $G_{s+1}$ is trivial, we get for all $g_{1}, \ldots, g_{s} \in G$ that

$$
\left[\left[\ldots\left[g_{1}, g_{2}\right], g_{3}\right], \ldots, g_{s}\right]^{r^{s}}=\left[\left[\ldots\left[g_{1}^{r}, g_{2}^{r}\right], g_{3}^{r}\right], \ldots, g_{s}^{r}\right] \in\left(G^{0} \Gamma\right)_{s} \text {. }
$$

Using this and because $G_{s}$ is Abelian and spanned by the elements $\left[\left[\ldots\left[g_{1}, g_{2}\right], g_{3}\right], \ldots, g_{s}\right]$, we deduce that for every $h \in G_{s}$ we have $h^{r} \in\left(G^{0} \Gamma\right)_{s}$. Since $G_{s}$ is connected for $s \geq 2$ it is divisible, hence the map $h \mapsto h^{r^{s}}$ is onto, and we conclude that $\left(G^{0} \Gamma\right)_{s}=G_{s}$.

\subsection{Nilsequences}

Following [3] we define:

Definition 3.1. If $X=G / \Gamma$ is an $s$-step nilmanifold, $F \in C(X)$, and $b \in G$, we call the sequence $\left(F\left(b^{n} \cdot e_{X}\right)\right)_{n \in \mathbb{N}}$ an $s$-step nilsequence (we omit the adjective "basic"). A 0-step nilsequence is a constant sequence. 


\section{NIKOS FRANTZIKINAKIS}

Remarks. - As remarked in Section 3.2, the set $Y=\overline{\left\{b^{n} \cdot e_{X}, n \in \mathbb{N}\right\}}$ is a sub-nilmanifold of $X$ that can be represented as $Y=H / \Delta$ for some closed subgroup $H$ of $G$ with $b \in H$. Thus, upon replacing $X$ with $Y$ we can assume that $b$ is an ergodic nilrotation of $X$.

- For every $x=g \Gamma \in X$, the sequence $\left(F\left(b^{n} x\right)\right)_{n \in \mathbb{N}}$ is a nilsequence, as it can be represented in the form $\left(F^{\prime}\left(b^{\prime n} \cdot e_{X}\right)\right)_{n \in \mathbb{N}}$ where $g^{\prime}:=g^{-1} b g$ and $F^{\prime}(x):=F(g x), x \in X$.

\subsubsection{Nilsequences of bounded complexity}

To every nilmanifold $X$ (equipped with a Riemannian metric) we associate a class of nilsequences of "bounded complexity" which will be used in the formulation of the inverse theorem in the next section.

Definition 3.2. Let $X=G / \Gamma$ be a nilmanifold. We let $\Psi_{X}$ be the set of nilsequences of the form $\left(\Psi\left(b^{n} x\right)\right)_{n \in \mathbb{N}}$ where $b \in G, x \in X$, and $\Psi \in \operatorname{Lip}(X)$ satisfies $\|\Psi\|_{\operatorname{Lip}(X)} \leq 1$.

Remark. Although $\Psi_{X}$ is not an algebra, there exists a nilmanifold $X^{\prime}$ (take $X^{\prime}=X \times X$ with a suitable Riemannian metric) such that $\Psi_{X^{\prime}}$ contains the sum and the product of any two elements of $\Psi_{X}$. We will often use this observation without further notice.

\subsubsection{Approximation by multiple-correlation sequences}

The next lemma will help us establish certain anti-uniformity properties of nilsequences that will be needed later. It is a consequence of [12, Proposition 2.4].

Lemma 3.3. Let $s \in \mathbb{N}$ and $X$ be an $s$-step nilmanifold. Then for every $\varepsilon, L>0$ there exists $M=M(\varepsilon, X, L)$ such that the following holds: If $\psi \in L \cdot \Psi_{X}$, then there exist a system $(Y, y, \mu, T)$ and functions $F_{0}, \ldots, F_{s} \in$ $L^{\infty}(\mu)$, all bounded by $M$, such that the sequence $(b(n))_{n \in \mathbb{N}}$, defined by

$$
b(n):=\int F_{0} \cdot T^{k_{1} n} F_{1} \cdot \ldots \cdot T^{k_{s} n} F_{s} d \mu, \quad n \in \mathbb{N},
$$

where $k_{j}:=\frac{(s+1) ! j}{j+1}$ for $j=1, \ldots, s$, satisfies

$$
\|\psi-b\|_{\infty} \leq \varepsilon
$$

Remark. Alternatively, we can use as approximants sequences of the form $b(n):=\lim _{M \rightarrow \infty} \mathbb{E}_{m \in[M]} a_{0}(m)$. $a_{1}\left(m+k_{1} n\right) \cdot \ldots \cdot a_{s}\left(m+k_{s} n\right), n \in \mathbb{N}$, where for $j=0, \ldots, s$ the sequences $a_{j} \in \ell^{\infty}(\mathbb{N})$ are defined by $a_{j}(m):=F_{j}\left(T^{m} y_{0}\right), m \in \mathbb{N}$, for suitable $y_{0} \in Y$.

Proof. Let $\varepsilon>0$. First note that since the space of functions on $\left(X, d_{X}\right)$ with Lipschitz constant at most $L$ is compact with respect to the $\|\cdot\|_{\infty}$-norm, we can cover this space by a finite number of $\|\cdot\|_{\infty}$-balls of radius at most $\varepsilon$. It follows from this that in order to verify the asserted approximation property, it suffices to verify the property for every fixed nilsequence $\psi$ without asking for additional uniformity properties for the $L^{\infty}(\mu)$ norms of the functions $F_{0}, \ldots, F_{s} \in L^{\infty}(\mu)$. This statement now follows immediately from [12, Proposition 2.4]. 


\subsubsection{Reduction of degree of nilpotency}

The next result will be used in the proof of the inverse theorem in the next section. It is a direct consequence of the constructions in [24, Section 7] and it is stated in a form equivalent to the one below in [40, Lemma 1.6.13] (for $\Phi_{1}=\Phi_{2}$ but the same argument works in the more general case):

Proposition 3.4 (Green, Tao [24]). For $s \geq 2$ let $X=G / \Gamma$ be an $s$-step nilmanifold. Then there exist an $(s-1)$-step nilmanifold $Y$ and $C=C(X)>0$ such that for all vertical nilcharacters $\Phi_{1}, \Phi_{2}$ of $X$ having the same frequency and satisfying $\left\|\Phi_{j}\right\|_{\operatorname{Lip}(X)} \leq 1, j=1,2$, every $b \in G$, and every $h \in \mathbb{N}$, the sequence $\left(\Phi_{1}\left(b^{n+h} \cdot e_{X}\right) \overline{\Phi_{2}\left(b^{n} \cdot e_{X}\right)}\right)_{n \in \mathbb{N}}$ is an $(s-1)$-step nilsequence in $C \cdot \Psi_{Y}$.

To illustrate this result by an example, take the 2-step nilsequence $\left(\mathrm{e}\left(n^{2} \alpha\right)\right)_{n \in \mathbb{N}}$ which can be defined by a vertical nilcharacter $\Phi$ on the Heisenberg nilmanifold. We take $\Phi_{1}=\Phi_{2}=\Phi$, then the difference operation results to the 1-step nilsequences $\left(\mathrm{e}\left(2 n h \alpha+h^{2} \alpha\right)\right)_{n \in \mathbb{N}}$ which can be represented as $\left(\Phi_{h}(n \beta)\right)_{n \in \mathbb{N}}, h \in \mathbb{N}$, where $\Phi_{h}: \mathbb{T} \rightarrow \mathbb{C}$ is defined by $\Phi_{h}(t):=\mathrm{e}\left(h^{2} \alpha\right) \mathrm{e}(t), h \in \mathbb{N}$, and $\beta:=2 h \alpha$.

\section{$4 U^{S}(\mathbf{I})$-inverse theorem for ergodic sequences}

Henceforth, we assume that $\mathbf{I}=\left(I_{N}\right)_{N \in \mathbb{N}}$ is a sequence of intervals with $\left|I_{N}\right| \rightarrow \infty$.

\subsection{Statement of the inverse theorem}

The goal of this section is to prove the following inverse theorem:

Theorem 4.1. Let $s \in \mathbb{N}$ and suppose that the sequence $a \in \ell^{\infty}(\mathbb{N})$ is ergodic for Cesàro averages on the sequence of intervals $\mathbf{I}=\left(I_{N}\right)_{N \in \mathbb{N}}$. If $\|a\|_{U^{s}(\mathbf{I})}>0$, then there exist an $(s-1)$-step nilsequence $\phi$ and an $(s-2)$-step nilmanifold $Y$ such that

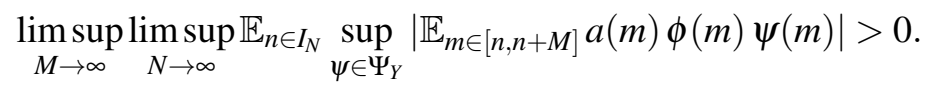

Remarks. • A variant for logarithmic averages also holds; one needs to assume ergodicity for logarithmic averages and replace $\|a\|_{U^{s+1}(\mathbf{I})}$ with $\|a\|_{U_{\log }^{s+1}(\mathbf{I})}$ and $\mathbb{E}_{n \in I_{N}}$ with $\mathbb{E}_{n \in I_{N}}^{\log }$.

- The ergodicity assumption is necessary. Indeed, if $a(n):=\sum_{k=1}^{\infty} \mathrm{e}\left(n \alpha_{k}\right) \mathbf{1}_{\left[k^{2},(k+1)^{2}\right)}(n), n \in \mathbb{N}$, where the sequence of real numbers $\left(\alpha_{k}\right)_{k \in \mathbb{N}}$ is chosen appropriately, then for every 1-step nilsequence $\phi$ we have

$$
\lim _{M \rightarrow \infty} \limsup _{N \rightarrow \infty} \mathbb{E}_{n \in[N]}\left|\mathbb{E}_{m \in[n, n+M]} a(m) \phi(m)\right|=0 .
$$

On the other hand for $\mathbf{I}=([N])_{N \in \mathbb{N}}$ we have $\|a\|_{U^{2}(\mathbf{I})}=1$.

- For general (not necessarily ergodic) sequences $a \in \ell^{\infty}(\mathbb{N})$ it can be shown (as in [42, Section 4]) that $\|a\|_{U^{s}(\mathbf{I})}=0$ holds if and only if for every $(s-1)$-step nilmanifold $Y$ we have

$$
\lim _{M \rightarrow \infty} \limsup _{N \rightarrow \infty} \mathbb{E}_{n \in I_{N}} \sup _{\psi \in \Psi_{Y}}\left|\mathbb{E}_{m \in[n, n+M]} a(m) \cdot \psi(m)\right|=0
$$




\section{NIKOS FRANTZIKINAKIS}

In particular for $s=2$ and $\mathbf{I}=([N])_{N \in \mathbb{N}}$, we get that $\|a\|_{U^{2}(\mathbf{I})}=0$ if and only if

$$
\lim _{M \rightarrow \infty} \limsup _{N \rightarrow \infty} \mathbb{E}_{n \in[N]} \sup _{t}\left|\mathbb{E}_{m \in[n, n+M]} a(m) \cdot \mathrm{e}(m t)\right|=0 .
$$

Despite its apparent simplicity, this condition is very hard to verify for particular arithmetic sequences, and it is still unknown for the Liouville and the Möbius function.

\subsection{Sketch of proof for $s=2$ versus $s>2$}

The proof of Theorem 4.1 is rather simple for $s=2$; we sketch it in order to motivate and explain some of the maneuvers needed in the general case. The argument proceeds as follows:

- We first use ergodicity of the sequence $(a(n))_{n \in \mathbb{N}}$ in order to establish the identity

$$
\|a\|_{U^{2}(\mathbf{I})}^{4}=\lim _{H \rightarrow \infty} \mathbb{E}_{h \in[H]}\left|\mathbb{E}_{n \in \mathbf{I}} a(n+h) \cdot \overline{a(n)}\right|^{2} .
$$

Using this identity and our assumption $\|a\|_{U^{2}(\mathbf{I})}>0$, we deduce that

$$
\lim _{H \rightarrow \infty} \mathbb{E}_{h \in[H]}\left(\mathbb{E}_{n \in \mathbf{I}} a(n+h) \cdot \overline{a(n)} \cdot A(h)\right)>0,
$$

where

$$
A(h):=\mathbb{E}_{n \in \mathbf{I}} \overline{a(n+h)} \cdot a(n), \quad h \in \mathbb{N} .
$$

This step generalizes straightforwardly when $s>2$ and gives relation (13) below.

- We can decompose the (positive definite) sequence $(A(n))_{n \in \mathbb{N}}$ into a structured component which is a trigonometric polynomial sequence and an error term which is small in uniform density. Hence, we can assume that (9) holds when $A(n)=\mathrm{e}(n t), n \in \mathbb{N}$, for some $t \in \mathbb{R}$. The use of an infinitary decomposition result is crucial in order to get for $s=2$ an inverse condition that does not involve a supremum in the inner-most average and for $s \geq 3$ an inverse condition that involves a supremum over $(s-2)$-step (and not $(s-1)$-step) nilsequences of bounded complexity. The appropriate decomposition result when $s \geq 3$ is Proposition 4.4 which is proved using deep results from ergodic theory (the main ingredient is Theorem 2.6). Since in this more complicated setup we cannot later on utilize simple identities that linear exponential sequences satisfy, we take particular care to use as structured components sequences which have a very convenient (though seemingly complicated) form.

- After interchanging the averages over $h$ and $n$ in (9), we take absolute values, and deduce that

$$
\underset{H \rightarrow \infty}{\limsup } \limsup _{N \rightarrow \infty} \mathbb{E}_{n \in I_{N}}\left|\mathbb{E}_{h \in[n, n+H]} a(h) \cdot \mathrm{e}(h t)\right|>0,
$$

which immediately implies the conclusion of Theorem 4.1 when $s=2$. This step is harder when $s>2$ and two additional maneuvers are needed (described in Steps 3 and 4 in the proof of Theorem 4.1). One key idea is to introduce an additional short range average that allows us to replace some unwanted expressions with $(s-2)$ nilsequences. This part of the argument uses the 
finitary decomposition result of Proposition 4.6 which is the reason why we get an inverse condition involving a sup over all $(s-2)$-nilsequences of bounded complexity. Another idea needed is to use Proposition 3.4 in order to remove an unwanted supremum over a parameter $h \in \mathbb{N}$; not doing so would cause serious problems later on when we try to verify the inverse condition for the class of multiplicative functions we are interested in.

We give the details of the proof of Theorem 4.1 in the next subsections.

\subsection{Uniformity estimates}

We will use the next estimate in the proof of Lemma 4.3, which in turn will be used in the proof of Proposition 4.6.

Lemma 4.2. Let $s \geq 2, M \in \mathbb{N}$, and $a_{\varepsilon}: \mathbb{Z}_{M} \rightarrow \mathbb{C}, \varepsilon \in\left[[s]^{*}\right.$. Then

$$
\mathbb{E}_{m \in \mathbb{Z}_{M}}\left|\mathbb{E}_{\underline{h} \in \mathbb{Z}_{M}^{s}} \prod_{\varepsilon \in \llbracket s \rrbracket^{*}} a_{\varepsilon}(m+\varepsilon \cdot \underline{h})\right| \leq \prod_{\varepsilon \in \llbracket s \rrbracket^{*}}\left\|a_{\varepsilon}\right\|_{U^{s}\left(\mathbb{Z}_{M}\right)} .
$$

Proof. Notice that the left hand side is equal to

$$
\mathbb{E}_{m \in \mathbb{Z}_{M}}\left(\mathbb{E}_{\underline{h} \in \mathbb{Z}_{M}^{s}} \prod_{\varepsilon \in \llbracket s \rrbracket} a_{\varepsilon}(m+\varepsilon \cdot \underline{h})\right),
$$

where $a_{\underline{0}}: \mathbb{Z}_{M} \rightarrow \mathbb{C}$ is defined by

$$
a_{\underline{0}}(m):=e^{-i \phi_{m}}, \quad \phi_{m}:=\arg \left(\mathbb{E}_{\underline{h} \in \mathbb{Z}_{M}^{s}} \prod_{\varepsilon \in \llbracket s]^{*}} a_{\varepsilon}(m+\varepsilon \cdot \underline{h})\right) .
$$

Since $\left\|a_{0}\right\|_{\infty} \leq 1$, the claimed estimate follows from the Gowers-Cauchy-Schwarz inequality [20, Lemma 3.8].

We use this lemma in order to deduce a similar estimate for non-periodic sequences:

Lemma 4.3. Let $s \geq 2, M \in \mathbb{N}$, and $a_{\varepsilon}:[(s+1) M] \rightarrow \mathbb{C}, \varepsilon \in[[s]]^{*}$, be bounded by 1 . Then

$$
\mathbb{E}_{m \in[M]}\left|\mathbb{E}_{\underline{h} \in[M]^{s}} \prod_{\varepsilon \in \llbracket s \rrbracket^{*}} a_{\varepsilon}(m+\varepsilon \cdot \underline{h})\right| \leq C_{s}\left(\min _{\varepsilon \in \llbracket s \rrbracket^{*}}\left\|a_{\mathcal{\varepsilon}}\right\|_{U^{s}\left(\mathbb{Z}_{(s+1) M}\right)}^{\frac{1}{s+1}}+\frac{1}{M}\right)
$$

where $C_{s}:=(s+1)^{s+1}\left((2 s)^{s}+1\right)$.

Proof. Let $\widetilde{M}:=(s+1) M$. We first reduce matters to estimating a similar average over $\mathbb{Z}_{\widetilde{M}}$. Let $\underline{h}=\left(h_{1}, \ldots, h_{s}\right)$ and notice that the average in (10) is bounded by $(s+1)^{s+1}$ times

$$
\mathbb{E}_{m \in \mathbb{Z}_{\tilde{M}}}\left|\mathbb{E}_{\underline{h} \in \mathbb{Z}_{\tilde{M}}^{s}} \prod_{j=1}^{s} \mathbf{1}_{[M]}\left(h_{j}\right) \cdot \prod_{\varepsilon \in \llbracket s \rrbracket^{*}} a_{\varepsilon}(m+\varepsilon \cdot \underline{h})\right|
$$

where the sums $m+\varepsilon \cdot \underline{h}$ are taken $(\bmod \widetilde{M})$. 


\section{NIKOS FRANTZIKINAKIS}

Next, we reduce matters to estimating a similar average that does not contain the indicator functions. Let $R$ be an integer that will be specified later and satisfies $0<R<M / 2$. We define the "trapezoid function" $\phi$ on $\mathbb{Z}_{\widetilde{M}}$ so that $\phi(0)=0, \phi$ increases linearly from 0 to 1 on the interval $[0, R], \phi(r)=1$ for $R \leq r \leq M-R, \phi$ decreases linearly from 1 to 0 on $[M-R, M]$, and $\phi(r)=0$ for $M<r<\widetilde{M}$.

After telescoping, we see that the absolute value of the difference between the average (11) and the average

$$
\mathbb{E}_{m \in \mathbb{Z}_{\widetilde{M}}}\left|\mathbb{E}_{\underline{h} \in \mathbb{Z}_{\tilde{M}}^{s}} \prod_{j=1}^{s} \phi\left(h_{j}\right) \cdot \prod_{\varepsilon \in \llbracket s]^{*}} a_{\varepsilon}(m+\varepsilon \cdot \underline{h})\right|
$$

is bounded by $2 s R / \widetilde{M}$. Moreover, it is classical that

$$
\|\widehat{\phi}\|_{l^{1}\left(\mathbb{Z}_{\tilde{M}}\right)} \leq \frac{2 M}{R} \leq \frac{\widetilde{M}}{R}
$$

and thus (12) is bounded by

$$
\frac{\widetilde{M}^{s}}{R^{s}} \max _{\xi_{1}, \ldots, \xi_{s} \in \mathbb{Z}_{\tilde{M}}} \mathbb{E}_{m \in \mathbb{Z}_{\widetilde{M}}}\left|\mathbb{E}_{\underline{h} \in \mathbb{Z}_{\widetilde{M}}^{s}} \prod_{j=1}^{s} \mathrm{e}\left(h_{j} \xi_{j} / \widetilde{M}\right) \cdot \prod_{\varepsilon \in\left[s \rrbracket^{*}\right.} a_{\varepsilon}(m+\varepsilon \cdot \underline{h})\right| .
$$

For $j=1, \ldots, s$, let $\left.\varepsilon_{j} \in[s]\right]^{*}$ be the element that has 1 in the $j$-th coordinate and 0 's elsewhere. Upon replacing $a_{\varepsilon_{j}}(n)$ with $a_{\varepsilon_{j}}(n) \mathrm{e}\left(-n \xi_{j} / \widetilde{M}\right), j=1, \ldots, s$, and $a_{(1, \ldots, 1)}(n)$ with $a_{(1, \ldots, 1)}(n) \mathrm{e}\left(n\left(\xi_{1}+\right.\right.$ $\left.\left.\cdots+\xi_{s}\right) / \widetilde{M}\right)$, and leaving all other sequences unchanged, the $U^{s}\left(\mathbb{Z}_{\widetilde{M}}\right)$-norm of all sequences remains unchanged (we use here that $s \geq 2$ ) and the term $\prod_{j=1}^{s} \mathrm{e}\left(h_{j} \xi_{j} / \widetilde{M}\right)$ disappears. We are thus left with estimating the average

$$
\mathbb{E}_{m \in \mathbb{Z}_{\tilde{M}}}\left|\mathbb{E}_{\underline{h} \in \mathbb{Z}_{\tilde{M}}^{s}} \prod_{\varepsilon \in[s] \rrbracket^{*}} a_{\varepsilon}(m+\varepsilon \cdot \underline{h})\right| .
$$

Since the sequences $a_{\varepsilon}, \varepsilon \in\left[[s]^{*}\right.$, are bounded by 1 , Lemma 4.2 implies that the last expression is bounded by

$$
U:=\min _{\varepsilon \in[s]]^{*}}\left\|a_{\varepsilon}\right\|_{U^{s}\left(\mathbb{Z}_{\tilde{M}}\right)}
$$

Combining the preceding estimates, we get that the average in the statement is bounded by $(s+1)^{s+1}$ times

$$
\frac{2 s R}{\widetilde{M}}+\frac{\widetilde{M}^{s}}{R^{s}} U
$$

Choosing $R:=\left\lfloor U^{\frac{1}{s+1}} \widetilde{M} /(4 s)\right\rfloor+1$ (then $R \leq M / 2$ for $M \geq 5$ ) we get that the last quantity is bounded by

$$
\left((4 s)^{s}+1\right) U^{\frac{1}{s+1}}+\frac{2}{M}
$$

when $M \geq 5$. When $M \leq 4$ the asserted estimate is trivial, completing the proof. 


\section{ERgodicity of the Liouville System Implies the Chowla Conjecture}

\subsection{Two decompositions}

We will use the following infinitary decomposition result which is proved using tools from ergodic theory.

Proposition 4.4. Let $s \in \mathbb{N}$ and suppose that the sequence $a \in \ell^{\infty}(\mathbb{N})$ is ergodic for Cesàro averages on the sequence of intervals $\mathbf{I}=\left(I_{N}\right)_{N \in \mathbb{N}}$. Then for every $s \in \mathbb{N}$ the sequence $A: \mathbb{N}^{s} \rightarrow \mathbb{C}$ defined by

$$
A(\underline{h}):=\mathbb{E}_{n \in \mathbf{I}} \prod_{\varepsilon \in \llbracket s \rrbracket} \mathcal{e}^{|\varepsilon|} a(n+\varepsilon \cdot \underline{h}), \quad \underline{h} \in \mathbb{N}^{s},
$$

admits a decomposition of the form $A=A_{\mathrm{st}}+A_{\mathrm{er}}$ such that

1. $A_{\mathrm{st}}: \mathbb{N}^{s} \rightarrow \mathbb{C}$ is a uniform limit of sequences of the form

$$
\underline{h} \mapsto \int A_{s t, x}(\underline{h}) d \mu(x), \quad \underline{h} \in \mathbb{N}^{s},
$$

where the integration takes place on a probability space $(X, X, \mu)$, and for $x \in X$ the sequence $A_{s t, x}: \mathbb{N}^{s} \rightarrow \mathbb{C}$ is defined by

$$
A_{s t, x}(\underline{h}):=\mathbb{E}_{n \in \mathbf{I}} \prod_{\varepsilon \in \llbracket s \rrbracket} \mathrm{e}^{|\varepsilon|} \phi_{x}(n+\varepsilon \cdot \underline{h}), \quad \underline{h} \in \mathbb{N}^{s},
$$

where the sequence $\phi_{x}: \mathbb{N} \rightarrow \mathbb{C}$ is an s-step nilsequence with $\left\|\phi_{x}\right\|_{\infty} \leq\|a\|_{\infty}$, and for $n \in \mathbb{N}$ the map $x \mapsto \phi_{x}(n)$ is $\mu$-measurable; and

2. $\lim _{M \rightarrow \infty} \mathbb{E}_{\underline{h} \in[M]^{s}}\left|A_{\mathrm{er}}(\underline{h})\right|=0$.

Remarks. - The ergodicity assumption in this statement is a convenience; we can prove a similar statement without it by using the decomposition result in [7, Proposition 3.1] in place of Theorem 2.6.

- It can be shown that the sequence $A_{\mathrm{st}}$ is a uniform limit of $s$-step nilsequences in $s$ variables, but such a decomposition result is less useful for our purposes.

Proof. Let $(X, X, \mu, T)$ be the ergodic system and $F \in L^{\infty}(\mu)$ be the function associated to $(a(n))_{n \in \mathbb{N}}$ and $\mathbf{I}$ by the correspondence principle of Proposition 2.3. Then

$$
A(\underline{h}):=\int \prod_{\varepsilon \in \llbracket s \rrbracket} \mathrm{C}^{|\varepsilon|} T^{\mathcal{\varepsilon} \cdot \underline{h}} F d \mu, \quad \underline{h} \in \mathbb{N}^{s} .
$$

We set

$$
A_{\mathrm{st}}(\underline{h}):=\int \prod_{\varepsilon \in \llbracket s \rrbracket} \mathrm{C}^{|\varepsilon|} T^{\mathcal{E} \cdot \underline{h}} F_{\mathrm{st}} d \mu, \quad \underline{h} \in \mathbb{N}^{s},
$$

where $F_{\mathrm{st}}:=\mathbb{E}\left(F \mid z_{s}\right)$ is the orthogonal projection of $F$ onto $L^{2}\left(z_{s}\right)$ and $z_{s}$ is the $\sigma$-algebra defined in Section 2.4. Furthermore, we let

$$
A_{\mathrm{er}}:=A-A_{\mathrm{st}} .
$$




\section{NIKOS FRANTZIKINAKIS}

We first deal with the sequence $A_{\mathrm{er}}$. It follows from [28, Theorem 13.1] that if $F_{\varepsilon} \in L^{\infty}(\mu)$ for all $\varepsilon \in\left[[s]\right.$ and $\left\|F_{\varepsilon}\right\|_{s+1, T}=0$ for some $\varepsilon \in[[s]$, then

$$
\lim _{M \rightarrow \infty} \mathbb{E}_{\underline{h} \in[M]^{s}}\left|\int \prod_{\varepsilon \in[s]} T^{\mathcal{E} \cdot \underline{h}} F_{\varepsilon} d \mu\right|=0
$$

Using this, telescoping, and since $F-F_{\mathrm{st}} \perp L^{2}\left(Z_{s}\right)$ implies $\left\|F-F_{\mathrm{st}}\right\|_{s+1, T}=0$, we deduce that

$$
\lim _{M \rightarrow \infty} \mathbb{E}_{\underline{h} \in[M]^{s}}\left|A_{\text {er }}(\underline{h})\right|=0 .
$$

Next we establish the asserted structural property of the sequence $A_{\mathrm{st}}$. Theorem 2.6 gives that the system $\left(X, Z_{s}, \mu, T\right)$ is an inverse limit of $s$-step nilsystems. It follows from this that the sequence $A_{\text {st }}$ is a uniform limit of sequences of the form

$$
\underline{h} \mapsto \int \prod_{\varepsilon \in[s \rrbracket} \mathcal{e}^{|\varepsilon|} \Phi\left(b^{\varepsilon \cdot \underline{h}} x\right) d m_{X}, \quad \underline{h} \in \mathbb{N}^{s},
$$

where $X=G / \Gamma$ is an $s$-step nilmanifold, $b \in G, m_{X}$ is the normalized Haar measure of $X$, and $\Phi \in C(X)$ satisfies

$$
\|\Phi\|_{\infty} \leq\left\|F_{s t}\right\|_{\infty} \leq\|F\|_{\infty}=\|a\|_{\infty} .
$$

As remarked in Section 3.1, for every $\underline{h} \in \mathbb{N}^{s}$ the limit $\mathbb{E}_{n \in \mathbf{I}} \prod_{\varepsilon \in \llbracket s \rrbracket} \mathrm{e}^{|\varepsilon|} \Phi\left(b^{n+\varepsilon \cdot \underline{h}} x\right)$ exists. Using this property, the bounded convergence theorem, and the preservation of $m_{X}$ by left translation by $b^{n}, n \in \mathbb{N}$, we get

$$
\int \prod_{\varepsilon \in \llbracket s \rrbracket} \mathrm{e}^{|\varepsilon|} \Phi\left(b^{\varepsilon \cdot \underline{h}} x\right) d m_{X}=\int \mathbb{E}_{n \in \mathbf{I}} \prod_{\varepsilon \in \llbracket s \rrbracket} \mathrm{e}^{|\varepsilon|} \Phi\left(b^{n+\varepsilon \cdot \underline{h}} x\right) d m_{X}=\int \mathbb{E}_{n \in \mathbf{I}} \prod_{\varepsilon \in \llbracket s] \rrbracket} \mathrm{e}^{|\varepsilon|} \phi_{x}(n+\varepsilon \cdot \underline{h}) d m_{X},
$$

where for $x \in X$ we let $\phi_{x}(n):=\Phi\left(b^{n} x\right), n \in \mathbb{N}$. Note that $\left(\phi_{x}(n)\right)_{n \in \mathbb{N}}$ is an $s$-step nilsequence for every $x \in X,\left\|\phi_{x}\right\|_{\infty} \leq\|\Phi\|_{\infty} \leq\|a\|_{\infty}$, and for fixed $n \in \mathbb{N}$ the map $x \mapsto \phi_{x}(n)=\Phi\left(b^{n} x\right)$ is $m_{X}$-measurable. This completes the proof.

The next result is proved in [22] using the finitary inverse theorem for the Gowers uniformity norms in [26]. Recall that for a given nilmanifold $X$ the set $\Psi_{X}$ was defined in Section 3.4.1.

Theorem 4.5 (Green, Tao [22, Proposition 2.7]). Let $\delta>0$ and $s \geq 2$ be an integer. Then there exist a positive number $L:=L(\delta, s)$ and an $(s-1)$-step nilmanifold $X=X(\delta, s)$ such that the following holds: For every large enough $M \in \mathbb{N}$, every $a:[M] \rightarrow \mathbb{C}$ that is bounded by 1 , admits a decomposition $a=a_{M, \mathrm{st}}+a_{M, \text { un }}$ such that

1. $a_{M, \mathrm{st}}$ is an (s-1)-step nilsequence in $L \cdot \Psi_{X}$ and $\left\|a_{N, \mathrm{st}}\right\|_{\infty} \leq 4$; and

2. $\left\|a_{M, u n}\right\|_{U^{s}\left(\mathbb{Z}_{M}\right)} \leq \delta$. 
Remark. The proof in [22] is given for sequences with values in $[0,1]$, the stated result follows since any sequence with values on the unit disc is a complex linear combination of four sequences with values in $[0,1]$. Moreover, the result in [22] is given for the $U^{s}[M]$ norms, the stated version follows since for large enough $M \in \mathbb{N}$ the $U^{s}\left(\mathbb{Z}_{M}\right)$ and $U^{s}[M]$ norms are comparable (see [14, Lemma A.4]). Lastly, the statement in [22] contains a third term that is small in $L^{2}[M]$, this term has been absorbed in the $a_{M \text {,un }}$ term in our statement.

We will combine Lemma 4.3 and Theorem 4.5 in order to establish the following finitary decomposition result:

Proposition 4.6. For every $\varepsilon>0$ and $s \geq 2$ there exist $C=C(\varepsilon, s)>0$ and an $(s-1)$-step nilmanifold $Y=Y(\varepsilon, s)$ such that the following holds: For every large enough $M \in \mathbb{N}$ and every $a:[(s+1) M] \rightarrow \mathbb{C}$ that is bounded by 1 , the sequence $A:[M] \rightarrow \mathbb{C}$ defined by

$$
A(m):=\mathbb{E}_{\underline{h} \in[M]^{s}} \prod_{\varepsilon \in[s]^{*}} \mathcal{e}^{|\varepsilon|} a(m+\varepsilon \cdot \underline{h}), \quad m \in[M],
$$

admits a decomposition of the form $A=A_{M, \mathrm{st}}+A_{M, \mathrm{er}}$ such that

1. $A_{M, \mathrm{st}}(m):=\mathbb{E}_{\underline{h} \in[M]^{s}} \psi_{M, \underline{h}}(m), m \in[M]$, where $\psi_{M, \underline{h}} \in C \cdot \Psi_{Y}$ for all $\underline{h} \in[M]^{s}$; and

2. $\mathbb{E}_{m \in[M]}\left|A_{M, \text { er }}(m)\right| \leq \varepsilon$.

Proof. Let $\varepsilon>0$ and $s \geq 2$. We use the decomposition result of Theorem 4.5 for $\delta=\delta(\varepsilon, s)$ to be determined momentarily. We get an $(s-1)$-step nilmanifold $X=X(\delta, s)$ an $L=L(\delta, s)>0$, such that for every large enough $M \in \mathbb{N}$ we have a decomposition $a(n)=a_{M, \text { st }}(n)+a_{M \text {, un }}(n), n \in[(s+1) M]$, where $a_{M, \mathrm{st}} \in L \cdot \Psi_{X},\left\|a_{N, \mathrm{st}}\right\|_{\infty} \leq 4$, and $\left\|a_{M, \text { un }}\right\|_{U^{s}\left(\mathbb{Z}_{(s+1) M}\right)} \leq \delta$.

We let

$$
A_{M, \mathrm{st}}(m):=\mathbb{E}_{\underline{h} \in[M]^{s}} \prod_{\varepsilon \in \llbracket s]^{*}} \mathrm{e}^{|\varepsilon|} \psi_{M}(m+\varepsilon \cdot \underline{h}), \quad m \in[M],
$$

where $\psi_{M}:=a_{M, \text { st }}$, and

$$
A_{M, \mathrm{er}}:=A-A_{M, \mathrm{st}} .
$$

Using Lemma 4.3 and telescoping, we deduce that if $\delta$ is sufficiently small, depending on $\varepsilon$ and $s$ only, then $A_{M \text {,er }}$ satisfies (ii).

It remains to deal with the term $A_{M \text {,st }}$. Since $\psi_{M} \in L \cdot \Psi_{X}$, there exists an $(s-1)$-step nilmanifold $Y=Y(\varepsilon, s)$ (take $Y:=X^{2^{s}-1}$ with the product metric) and a constant $C=C(\delta, s)>0$ such that for every $M \in \mathbb{N}$ and $\underline{h} \in[M]^{s}$ the sequence $\psi_{M, \underline{h}}: \mathbb{N} \rightarrow \mathbb{C}$, defined by $\psi_{M, \underline{h}}(m):=\prod_{\varepsilon \in \llbracket s \rrbracket^{*}} \mathrm{C}^{|\varepsilon|} \psi_{M}(m+\varepsilon \cdot \underline{h}), m \in \mathbb{N}$, is in $C \cdot \Psi_{Y}$. This completes the proof.

\subsection{Proof of Theorem 4.1}

Let $s \geq 2$. Without loss of generality we can assume that $\|a\|_{\infty} \leq 1$. 


\section{NIKOS FRANTZIKINAKIS}

Step 1 (Using ergodicity). Using the ergodicity of $a(n)$ for Cesàro averages on $\mathbf{I}$ (this is the only place where we make essential use of ergodicity in the proof of Theorem 4.1) we get that

$$
\|a\|_{U^{s}(\mathbf{I})}^{2^{s}}=\mathbb{E}_{\underline{h} \in \mathbb{N}^{s-1}}\left|\mathbb{E}_{n \in \mathbf{I}} \prod_{\varepsilon \in \llbracket s-1 \rrbracket} \mathcal{C}^{|\varepsilon|} a(n+\varepsilon \cdot \underline{h})\right|^{2} .
$$

To see this note that

$$
\begin{aligned}
\|a\|_{U^{s}(\mathbf{I})}^{2^{s}} & =\mathbb{E}_{\underline{h} \in \mathbb{N}^{s}}\left(\mathbb{E}_{n \in \mathbf{I}} \prod_{\varepsilon \in \llbracket s \rrbracket} \mathcal{C}^{|\varepsilon|} a(n+\varepsilon \cdot \underline{h})\right) \\
& =\mathbb{E}_{\underline{h} \in \mathbb{N}^{s-1}} \mathbb{E}_{h \in \mathbb{N}}\left(\mathbb{E}_{n \in \mathbf{I}} \prod_{\varepsilon \in \llbracket s-1 \rrbracket} \mathcal{C}^{|\varepsilon|} a(n+\varepsilon \cdot \underline{h}+h) \cdot \prod_{\varepsilon \in \llbracket s-1 \rrbracket} \mathrm{e}^{|\varepsilon|} \overline{a(n+\varepsilon \cdot \underline{\boldsymbol{h}})}\right) \\
& =\mathbb{E}_{\underline{h} \in \mathbb{N}^{s-1}}\left(\mathbb{E}_{n \in \mathbf{I}} \prod_{\varepsilon \in \llbracket[s-1 \rrbracket} \mathcal{e}^{|\varepsilon|} a(n+\varepsilon \cdot \underline{h}) \cdot \mathbb{E}_{n \in \mathbf{I}} \prod_{\varepsilon \in \llbracket s-1 \rrbracket} \mathcal{C}^{|\varepsilon|} \overline{a(n+\varepsilon \cdot \underline{h})}\right) \\
& =\mathbb{E}_{\underline{h} \in \mathbb{N}^{s-1}}\left|\mathbb{E}_{n \in \mathbf{I}} \prod_{\varepsilon \in \llbracket s-1 \rrbracket} \mathcal{C}^{|\varepsilon|} a(n+\varepsilon \cdot \underline{h})\right|^{2},
\end{aligned}
$$

where the first identity follows from (4), the second follows from the remarks made in Section 2.5, and the third from our ergodicity assumption using identity (3).

Step 2 (Using an infinitary decomposition). Hence, if $\|a\|_{U^{s}(\mathbf{I})}>0$, then

$$
\mathbb{E}_{\underline{h} \in \mathbb{N}^{s-1}}\left(\mathbb{E}_{n \in \mathbf{I}} \prod_{\varepsilon \in \llbracket s-1 \rrbracket} \mathcal{C}^{|\varepsilon|} a(n+\varepsilon \cdot \underline{h}) \cdot A(\underline{h})\right)>0,
$$

where

$$
A(\underline{h}):=\mathbb{E}_{n^{\prime} \in \mathbf{I}} \prod_{\varepsilon \in \llbracket s-1 \rrbracket} \mathcal{C}^{|\varepsilon|+1} a\left(n^{\prime}+\varepsilon \cdot \underline{h}\right) .
$$

As remarked in Section 2.5, we can replace the average $\mathbb{E}_{\underline{h} \in \mathbb{N}^{s-1}}$ with $\lim _{H \rightarrow \infty} \mathbb{E}_{\underline{h} \in[H]^{s-1}}$. By Proposition 4.4 we have a decomposition

$$
A(\underline{h})=A_{\mathrm{st}}(\underline{h})+A_{\mathrm{er}}(\underline{h}), \quad \underline{h} \in \mathbb{N}^{s-1},
$$

where

1. $A_{\mathrm{st}}(\underline{h})$ is a uniform limit of sequences of the form

$$
\underline{h} \mapsto \int A_{\mathrm{st}, x}(\underline{h}) d \mu(x), \quad \underline{h} \in \mathbb{N}^{s-1},
$$

such that

$$
A_{\mathrm{st}, x}(\underline{h}):=\mathbb{E}_{n^{\prime} \in \mathbf{I}} \prod_{\varepsilon \in \llbracket s-1 \rrbracket} \mathrm{e}^{|\varepsilon|+1} \phi_{x}\left(n^{\prime}+\varepsilon \cdot \underline{h}\right), \quad \underline{h} \in \mathbb{N}^{s-1},
$$

where for $x \in X$ the sequence $\phi_{x}: \mathbb{N} \rightarrow \mathbb{C}$ is a basic $(s-1)$-step nilsequence with $\left\|\phi_{x}\right\|_{\infty} \leq\|a\|_{\infty} \leq 1$, and for $n \in \mathbb{N}$ the map $x \mapsto \phi_{x}(n)$ is $\mu$-measurable; and

2. $\lim _{M \rightarrow \infty} \mathbb{E}_{\underline{h} \in[M]^{s-1}}\left|A_{\mathrm{er}}(\underline{h})\right|=0$. 
Using uniform approximation and the second condition we deduce that

$$
\lim _{M \rightarrow \infty} \mathbb{E}_{\underline{h} \in[M]^{s-1}}\left(\mathbb{E}_{n \in \mathbf{I}} \prod_{\varepsilon \in \llbracket s-1 \rrbracket} \mathcal{C}^{|\varepsilon|} a(n+\varepsilon \cdot \underline{h}) \cdot \int A_{\text {st }, x}(\underline{h}) d \mu(x)\right)>0 .
$$

Hence,

$$
\lim _{M \rightarrow \infty} \int \mathbb{E}_{\underline{h} \in[M]^{s-1}}\left(\mathbb{E}_{n \in \mathbf{I}} \prod_{\varepsilon \in[s-1 \rrbracket} \mathcal{C}^{|\varepsilon|} a(n+\varepsilon \cdot \underline{h}) \cdot A_{\mathrm{st}, x}(\underline{h})\right) d \mu(x)>0 .
$$

Using Fatou's lemma we deduce that there exists an $x \in X$ such that

$$
\limsup _{M \rightarrow \infty}\left|\mathbb{E}_{\underline{h} \in[M]^{s-1}}\left(\mathbb{E}_{n \in \mathbf{I}} \prod_{\varepsilon \in \llbracket[s-1 \rrbracket} \mathcal{e}^{|\varepsilon|} a(n+\varepsilon \cdot \underline{h}) \cdot A_{\mathrm{st}, x}(\underline{h})\right)\right|>0
$$

Using the form of $A_{\mathrm{st}, x}$ and the fact that both limits $\mathbb{E}_{n \in \mathbf{I}} \cdots$ and $\mathbb{E}_{n^{\prime} \in \mathbf{I}} \cdots$ exist, we get that

$$
\limsup _{M \rightarrow \infty} \lim _{N \rightarrow \infty}\left|\mathbb{E}_{n, n^{\prime} \in I_{N}} a(n) \phi_{x}\left(n^{\prime}\right)\left(\mathbb{E}_{\underline{h} \in[M]^{s-1}} \prod_{\varepsilon \in[s-1]^{*}} \mathcal{e}^{|\varepsilon|}\left(a(n+\varepsilon \cdot \underline{h}) \cdot \overline{\phi_{x}\left(n^{\prime}+\varepsilon \cdot \underline{h}\right)}\right)\right)\right|>0 .
$$

Hence, renaming $\phi_{x}$ as $\phi$, we get that there exists an $(s-1)$-step nilsequence $\phi$ such that

$$
\limsup _{M \rightarrow \infty} \lim _{N \rightarrow \infty}\left|\mathbb{E}_{n, n^{\prime} \in I_{N}} a(n) \phi\left(n^{\prime}\right)\left(\mathbb{E}_{\underline{h} \in[M]^{s-1}} \prod_{\varepsilon \in[s-1]^{*}} \mathrm{e}^{|\varepsilon|} \tilde{a}_{n, n^{\prime}}(\varepsilon \cdot \underline{h})\right)\right|>0,
$$

where for $n, n^{\prime} \in \mathbb{N}$ the sequence $\left(\tilde{a}_{n, n^{\prime}}(k)\right)_{k \in \mathbb{N}}$ is defined by

$$
\tilde{a}_{n, n^{\prime}}(k):=a(n+k) \phi\left(n^{\prime}+k\right), \quad k \in \mathbb{N} .
$$

Step 3 (Using a finitary decomposition). Next, we shift the averages over $n$ and $n^{\prime}$ by $m \in \mathbb{N}$ and average over $m \in[M] .^{3}$ We deduce that

$$
\underset{M \rightarrow \infty}{\limsup \limsup } \mathbb{E}_{N \rightarrow \infty} \mathbb{n}^{\prime} \in I_{N}\left|\mathbb{E}_{m \in[M]} a(m+n) \phi\left(m+n^{\prime}\right) A_{M, n, n^{\prime}}(m)\right|=\delta>0,
$$

where for $M, n, n^{\prime} \in \mathbb{N}$ we let

$$
A_{M, n, n^{\prime}}(m):=\mathbb{E}_{\underline{h} \in[M]^{s-1}} \prod_{\varepsilon \in \llbracket s-1 \rrbracket^{*}} \mathcal{C}^{|\varepsilon|} \tilde{a}_{n, n^{\prime}}(m+\varepsilon \cdot \underline{h}), \quad m \in[M] .
$$

For $M, n, n^{\prime} \in \mathbb{N}$, we use Proposition 4.6 for $\varepsilon:=\delta / 3$ in order to decompose the finite sequence $A_{M, n, n^{\prime}}(m), m \in[M]$. We get that there exist $C=C(\delta, s)>0$, an $(s-2)$-step nilmanifold $Y=Y(\delta, s)$, and for large enough $M \in \mathbb{N}$ there exist $(s-2)$-step nilsequences $\psi_{M, n, n^{\prime}, \underline{h}} \in C \cdot \Psi_{Y}$, where $\underline{h} \in[M]^{s-1}$, $n, n^{\prime} \in \mathbb{N}$, such that

$$
\underset{M \rightarrow \infty}{\limsup \limsup } \mathbb{E}_{n \rightarrow \infty}{ }_{n, n^{\prime} \in I_{N}}\left|\mathbb{E}_{m \in[M]} a(m+n) \phi\left(m+n^{\prime}\right) \mathbb{E}_{\underline{h} \in[M]^{s-1}} \psi_{M, n, n^{\prime}, \underline{h}}(m)\right|>\delta / 2>0 .
$$

\footnotetext{
${ }^{3}$ We perform this maneuver in order to introduce a "small range parameter" $m \in[M]$ which will give rise to sequences in $m \in[M]$ for which finitary decomposition results are applicable.
} 
Hence,

$$
\underset{M \rightarrow \infty}{\limsup } \limsup _{N \rightarrow \infty} \mathbb{E}_{n, n^{\prime} \in I_{N}} \mathbb{E}_{\underline{h} \in[M]^{s-1}}\left|\mathbb{E}_{m \in[M]} a(m+n) \phi\left(m+n^{\prime}\right) \psi_{M, n, n^{\prime}, \underline{h}}(m)\right|>0,
$$

from which we deduce that

$$
\underset{M \rightarrow \infty}{\limsup \limsup } \mathbb{E}_{N \rightarrow \infty} \sin ^{\prime} \in I_{N} \sup _{\psi \in \Psi_{Y}}\left|\mathbb{E}_{m \in[M]} a(m+n) \phi\left(m+n^{\prime}\right) \psi(m)\right|>0 .
$$

This implies that (notice that $n \mapsto \psi(n+k)$ is in $\Psi_{Y}$ for every $k \in \mathbb{Z}$ )

$$
\limsup _{M \rightarrow \infty} \limsup _{N \rightarrow \infty} \mathbb{E}_{n \in I_{N}} \sup _{\psi \in \Psi_{Y}, h \in \mathbb{N}}\left|\mathbb{E}_{m \in[n, n+M]} a(m) \phi(m+h) \psi(m)\right|>0
$$

for some $(s-1)$-step nilsequence $\phi$.

Step 4 (Removing the sup over $h$ ). It remains to show that the supremum over $h \in \mathbb{N}$ can be removed. As we mentioned in Section 3.3, the linear span of vertical nilcharacters is dense in $C(X)$. Hence, we can assume that $\phi(n)=\Phi\left(b^{n} \cdot e_{X}\right), n \in \mathbb{N}$, for some $(s-1)$-step nilmanifold $X, b \in G$, and vertical nilcharacter $\Phi$ of $X$ with $\|\Phi\|_{\operatorname{Lip}(X)} \leq 1$. Although we cannot assume that $|\Phi(x)|=1$ for every $x \in X$, it is known (see the proof of [43, Proposition 5.6] or [26, Lemma 6.4]) that there exist $k \in \mathbb{N}$ and vertical nilcharacters $\Phi_{1}, \ldots, \Phi_{k}$ of $X$, which all have the same frequency as $\Phi$, and satisfy $\left\|\Phi_{j}\right\|_{\operatorname{Lip}(X)} \leq 1$ for $j=1, \ldots, k$ and $\left|\Phi_{1}(x)\right|^{2}+\cdots+\left|\Phi_{k}(x)\right|^{2}=1$ for every $x \in X$. For $j=1, \ldots, k$, we let $\phi_{j}(n):=\Phi_{j}\left(b^{n} \cdot e_{X}\right), n \in \mathbb{N}$. Then $\left|\phi_{1}(n)\right|^{2}+\cdots+\left|\phi_{k}(n)\right|^{2}=1$ for every $n \in \mathbb{N}$ and we deduce from this and (14) that for some $j_{0} \in\{1, \ldots, k\}$ we have

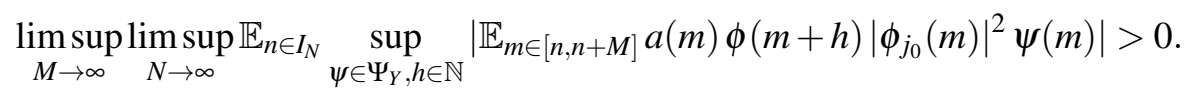

It follows from Proposition 3.4 that there exist an $(s-2)$-nilmanifold $\tilde{Y}$ and $\tilde{C}=\tilde{C}(Y)=\tilde{C}(\delta, s)>0$ such that for every $h \in \mathbb{N}$ the sequence $\left(\phi(n+h) \overline{\phi_{j_{0}}(n)}\right)_{n \in \mathbb{N}}$ is an $(s-2)$-step nilsequence in $\tilde{C} \cdot \Psi_{\tilde{Y}}$. Thus, upon enlarging the $(s-2)$-nilmanifold $Y$ in (15) the term $\phi(m+h) \overline{\phi_{j_{0}}(m)}$ can be absorbed in the supremum over $\psi \in \Psi_{Y}$. We deduce that

$$
\underset{M \rightarrow \infty}{\limsup \limsup } \mathbb{E}_{n \rightarrow \infty} \sup _{\psi \in \Psi_{Y}}\left|\mathbb{E}_{m \in[n, n+M]} a(m) \phi_{j_{0}}(m) \psi(m)\right|>0 .
$$

This completes the proof.

\section{$5 \quad U^{S}(\mathbf{I})$-uniformity for the Liouville function}

Our goal in this section is to prove Theorem 1.6. Note that this uniformity result combined with Theorem 1.7 gives Theorem 1.1 and combined with Theorem 1.8 gives Theorem 1.4. We present the proof of Theorem 1.6 for Cesàro averages but a similar argument also works for logarithmic averages; wherever needed we indicate which statements need to be modified for this purpose. 


\subsection{Sketch of the proof}

We proceed by induction as follows:

- For $s=2$ we get that Theorem 1.6 follows from the ergodicity of $f$ and Proposition 2.10 (the strong aperiodicity of $f$ is only used here). Assuming that $s \geq 2$ and $\|f\|_{U^{s}(\mathbf{I})}=0$, our goal then becomes to show that $\|f\|_{U^{s+1}(\mathbf{I})}=0$.

- We first use the inverse result of Theorem 4.1 in order to reduce matters to an orthogonality property of $f$ with nilsequences on typical short intervals (see Proposition 5.1). Essential use of ergodicity of $f$ is made here.

- The orthogonality property involves a fixed $s$-step nilsequence $\phi$ and a supremum over a set of $(s-1)$ step nilsequences of bounded complexity. If $\phi$ is an $(s-1)$-step nilsequence, then we are done by the induction hypothesis (the ergodicity of $f$ is used again here) via elementary estimates. If not, we reduce matters to the case where the $s$-step nilsequence $\phi$ is defined by a non-trivial vertical nilcharacter (see Proposition 5.3).

- We then use the orthogonality criterion of Lemma 5.5 (the multiplicativity of $f$ is only used here) to reduce matters to a purely dynamical statement about "irrational nilsequences" (see Proposition 5.6).

- Lastly, we verify the dynamical statement using elementary estimates, qualitative equidistribution results on nilmanifolds, and ideas motivated from [14].

\subsection{Step 1 (Setting up the induction and cases $s=1,2$ )}

We prove Theorem 1.6 by induction on $s \in \mathbb{N}$. We cover the cases $s=1$ and $s=2$ separately, partly because we want to show their relation to recently established results, but also because the inductive step $s \mapsto s+1$ is slightly different when $s \geq 2$.

For $s=1$ we have (recall that $\left.\mathbf{I}=\left(\left[N_{k}\right]\right)_{k \in \mathbb{N}}\right)$

$$
\|f\|_{U^{1}(\mathbf{I})}^{2}=\lim _{H \rightarrow \infty} \mathbb{E}_{h \in[H]}\left(\mathbb{E}_{n \in \mathbf{I}} f(n+h) \overline{f(n)}\right) \leq \limsup _{H \rightarrow \infty} \limsup _{N \rightarrow \infty} \mathbb{E}_{n \in[N]}\left|\mathbb{E}_{h \in[H]} f(n+h)\right|
$$

and the last limit is 0 by [35, Theorem A.1]. Note that this argument did not use our ergodicity assumption on $f$. Assuming ergodicity of $f$ for Cesàro averages on $\mathbf{I}$, then one simply notes that $\|f\|_{U^{1}(\mathbf{I})}=\left|\mathbb{E}_{n \in \mathbf{I}} f(n)\right|=0$.

For $s=2$, using our hypothesis that the sequence $(f(n))_{n \in \mathbb{N}}$ is ergodic for Cesàro averages on I we derive exactly as in the first step of the proof of Theorem 4.1 the identity

$$
\|f\|_{U^{2}(\mathbf{I})}^{4}=\lim _{H \rightarrow \infty} \mathbb{E}_{h \in[H]}\left|\mathbb{E}_{n \in \mathbf{I}} f(n+h) \cdot \overline{f(n)}\right|^{2} .
$$

This limit is 0 by Proposition 2.10 .

Suppose now that Theorem 1.6 holds for $s \geq 2$; in the remaining subsections we will show that it holds for $s+1$. 


\section{NIKOS FRANTZIKINAKIS}

\subsection{Step 2 (Using the inverse theorem)}

We start by using the inverse theorem proved in the previous section. It follows from Theorem 4.1 and Proposition 2.7 that in order to prove Theorem 1.6 it suffices to establish the following result:

Proposition 5.1. Let $s \geq 2$ and $f \in \mathcal{M}$ be a strongly aperiodic multiplicative function which is ergodic for Cesàro averages on the sequence of intervals $\mathbf{I}=\left(\left[N_{k}\right]\right)_{k \in \mathbb{N}}$. Then for every s-step nilsequence $\phi$ and every $(s-1)$-step nilmanifold $Y$, we have

$$
\lim _{M \rightarrow \infty} \limsup _{k \rightarrow \infty} \mathbb{E}_{n \in\left[N_{k}\right]} \sup _{\psi \in \Psi_{Y}}\left|\mathbb{E}_{m \in[n, n+M]} f(m) \phi(m) \psi(m)\right|=0
$$

Remark. - A variant for logarithmic averages also holds where one assumes ergodicity for logarithmic averages on $\mathbf{I}$ and replaces $\mathbb{E}_{n \in\left[N_{k}\right]}$ with $\mathbb{E}_{n \in\left[N_{k}\right]}^{\log }$. The proof is similar.

- If we remove the $\sup _{\psi \in \Psi_{Y}}$, then our proof works without an ergodicity assumption on $f$. This simpler result was also obtained recently in [11], and prior to this, related results were obtained in $[14,25]$. But none of these results allows to treat the more complicated setup with the supremum over the set $\Psi_{Y}$ and this is crucial for our purposes.

\subsection{Step 3 (Reduction to non-trivial nilcharacters)}

Since $\phi$ is an $s$-step nilsequence, there exist an $s$-step nilmanifold $X=G / \Gamma$, an ergodic nilrotation $b \in G$, and a function $\Phi \in C(X)$, such that $\phi(n)=\Phi\left(b^{n} \cdot e_{X}\right), n \in \mathbb{N}$. Since the linear span of vertical nilcharacters of $X$ are dense $C(X)$ (see Section 3.3) we can assume that $\Phi$ is a vertical nilcharacter of $X$.

If $\Phi$ is a trivial nilcharacter of $X$, then it factorizes through the nilmanifold

$$
X^{\prime}=G /\left(G_{s} \Gamma\right)=\left(G / G_{s}\right) /\left(\left(\Gamma \cap G_{s}\right) / G_{s}\right)
$$

The group $G / G_{s}$ is $(s-1)$-step nilpotent and $X^{\prime}$ is an $(s-1)$-step nilmanifold. Writing $b^{\prime}$ for the image of $b$ in $G / G_{s}$, we have that $\phi(n)=\Phi^{\prime}\left(b^{\prime n} \cdot e_{X^{\prime}}\right), n \in \mathbb{N}$, for some $\Phi^{\prime} \in C\left(X^{\prime}\right)$. We deduce that $\phi$ is an $(s-1)$-step nilsequence. Moreover, since the sequence $(f(n))_{n \in \mathbb{N}}$ is ergodic for Cesàro averages on $\mathbf{I}=\left(\left[N_{k}\right]\right)_{k \in \mathbb{N}}$, the induction hypothesis gives that $\|f\|_{U^{s}(\mathbf{I})}=0$. Hence, the following theorem (which does not require ergodicity assumptions) implies that the conclusion of Proposition 5.1 holds when the function $\Phi$ defining the nilsequence $\phi$ is a trivial nilcharacter of $X$ :

Lemma 5.2. Let $s \geq 2$ and $a \in \ell^{\infty}(\mathbb{N})$ be a sequence that admits correlations for Cesàro averages on the sequence of intervals $\mathbf{I}=\left(I_{N}\right)_{N \in \mathbb{N}}$. Suppose that $\|a\|_{U^{s}(\mathbf{I})}=0$. Then for every $(s-1)$-step nilsequence $\phi$ and every $(s-1)$-step nilmanifold $Y$, we have

$$
\lim _{M \rightarrow \infty} \limsup _{N \rightarrow \infty} \mathbb{E}_{n \in I_{N}} \sup _{\psi \in \Psi_{Y}}\left|\mathbb{E}_{m \in[n, n+M]} a(m) \phi(m) \psi(m)\right|=0 .
$$

Remark. A variant for logarithmic averages also holds where one replaces $\|a\|_{U^{s}(\mathbf{I})}$ with $\|a\|_{U_{\log }^{s}(\mathbf{I})}$ and $\mathbb{E}_{n \in I_{N}}$ with $\mathbb{E}_{n \in I_{N}}^{\log }$. The proof is similar. 


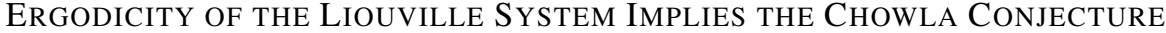

Proof. First notice that since every $(s-1)$-step nilsequence $\phi$ can be uniformly approximated by $(s-1)$ step nilsequences defined by functions with bounded Lipschitz norm, the sequence $\phi$ can be absorbed in the sup (upon enlarging the nilmanifold $Y$ ). Hence, we can assume that $\phi=1$.

By Lemma 3.3 it suffices to show that

$$
\lim _{M \rightarrow \infty} \limsup _{N \rightarrow \infty} \mathbb{E}_{n \in I_{N}}\left|\mathbb{E}_{m \in[M]} a(m+n) \int F_{0, M, n} \cdot T_{M, n}^{k_{1} m} F_{1, M, n} \cdot \ldots \cdot T_{M, n}^{k_{s-1} m} F_{S-1, M, n} d \mu_{M, n}\right|=0,
$$

where the integers $k_{1}, \ldots, k_{s-1}$ are arbitrary, and for $M, n \in \mathbb{N}$ we have that $\left(X_{M, n}, X_{M, n}, \mu_{M, n}, T_{M, n}\right)$ is a system and the functions $F_{0, M, n}, \ldots, F_{s, M, n} \in L^{\infty}\left(\mu_{M, n}\right)$ are bounded by 1 . In fact, we will show by induction on $s \in \mathbb{N}$ a stronger statement, namely, that if the functions and the sequence are as above and they are all bounded by 1 , then

$$
\underset{M \rightarrow \infty}{\limsup \limsup } \mathbb{E}_{n \rightarrow \infty}\left\|\mathbb{E}_{m \in[M]} a(m+n) T_{M, n}^{k_{1} m} F_{1, M, n} \cdots T_{M, n}^{k_{s-1} m} F_{s-1, M, n}\right\|_{L^{2}\left(\mu_{M, n}\right)} \leq 4\|a\|_{U^{s}(\mathbf{I})} .
$$

We implicitly assume that the $s=1$ case corresponds to an estimate where there are no functions on the left hand side. So in order to verify the base case, we need to show that

$$
\underset{M \rightarrow \infty}{\limsup } \limsup _{N \rightarrow \infty} \mathbb{E}_{n \in I_{N}}\left|\mathbb{E}_{m \in[M]} a(m+n)\right| \leq 4\|a\|_{U^{1}(\mathbf{I})} .
$$

To this end, we apply the van der Corput lemma for complex numbers. We get for all $M, R \in \mathbb{N}$ with $R \leq M$ and all $n \in \mathbb{N}$, that

$$
\left|\mathbb{E}_{m \in[M]} a(n+m)\right|^{2} \leq 4 \mathbb{E}_{r \in[R]}\left(1-r R^{-1}\right)\left(\Re\left(\mathbb{E}_{m \in[M]} a(m+n+r) \cdot \overline{a(m+n)}\right)+R^{-1}+R M^{-1}\right) .
$$

Hence,

$$
\begin{aligned}
& \underset{M \rightarrow \infty}{\limsup \limsup \left(\mathbb{E}_{n \in I_{N}}\left|\mathbb{E}_{m \in[M]} a(n+m)\right|\right)^{2} \leq} \\
& \qquad \underset{R \rightarrow \infty}{4 \limsup _{R \rightarrow \infty} \limsup _{M \rightarrow \infty}} \mathbb{E}_{r \in[R]}\left(1-r R^{-1}\right) \mathbb{E}_{m \in[M]} \Re\left(\mathbb{E}_{n \in \mathbf{I}} a(m+n+r) \cdot \overline{a(m+n)}\right)= \\
& \qquad \limsup _{R \rightarrow \infty} \mathbb{E}_{r \in[R]}\left(1-r R^{-1}\right) \Re\left(\mathbb{E}_{n \in \mathbf{I}} a(n+r) \cdot \overline{a(n)}\right) \leq \\
& \quad 4 \limsup _{R \rightarrow \infty} \mathbb{E}_{r \in[R]} \Re\left(\mathbb{E}_{n \in \mathbf{I}} a(n+r) \cdot \overline{a(n)}\right)=4\|a\|_{U^{1}(\mathbf{I})}^{2},
\end{aligned}
$$

where the last estimate holds because $\mathbb{E}_{r \in[R]}\left(1-r R^{-1}\right) \Re\left(\mathbb{E}_{n \in \mathbf{I}} a(n+r) \cdot \overline{a(n)}\right)$ is the Cesàro average of $\mathbb{E}_{r \in[R]} \Re\left(\mathbb{E}_{n \in \mathbf{I}} a(n+r) \cdot \overline{a(n)}\right)$ with respect to $R$. Hence, the asserted estimate holds.

Suppose now that the estimate (16) holds for $s-1 \in \mathbb{N}$, where $s \geq 2$, we will show that it holds for $s$. We apply the van der Corput lemma in the Hilbert space $L^{2}\left(\mu_{M, n}\right)$ and then use the Cauchy-Schwarz inequality. We get for all $M, R \in \mathbb{N}$ with $R \leq M$ and all $n \in \mathbb{N}$, that

$$
\begin{aligned}
& \left\|\mathbb{E}_{m \in[M]} a(m+n) T_{M, n}^{k_{1} m} F_{1, M, n} \cdots T_{M, n}^{k_{s-1} m} F_{S-1, M, n}\right\|_{L^{2}\left(\mu_{M, n}\right)}^{2} \leq \\
& \quad 4 \mathbb{E}_{r \in[R] \|}\left\|\mathbb{E}_{m \in[M]} a(m+n+r) \cdot \overline{a(m+n)} T_{M, n}^{\tilde{k}_{1} m} \tilde{F}_{1, M, n, r} \cdots T_{M, n}^{\tilde{k}_{s-1} m} \tilde{F}_{S-2, M, n, r}\right\|_{L^{2}\left(\mu_{M, n}\right)}+R^{-1}+R M^{-1},
\end{aligned}
$$




\section{NIKOS FRANTZIKINAKIS}

where $\tilde{F}_{j, M, n, r}:=T_{M, n}^{k_{j} r} F_{j, M, n} \cdot \overline{F_{j, M, n}}, \tilde{k}_{j}:=k_{j}-k_{s-1}$, for $j=1, \ldots, s-2$.

Hence, the square of the left hand side in (16) is bounded by

$$
\begin{aligned}
& \quad 4 \limsup _{R \rightarrow \infty} \mathbb{E}_{r \in[R]} \limsup \limsup _{M \rightarrow \infty} \mathbb{E}_{n \in I_{N}} \\
& \qquad\left\|\mathbb{E}_{m \in[M]} a(m+n+r) \cdot \overline{a(m+n)} T_{M, n}^{\tilde{k}_{1} m} \tilde{F}_{1, M, n, r} \cdots T_{M, n}^{\tilde{k}_{s-1} m} \tilde{F}_{S-2, M, n, r}\right\|_{L^{2}\left(\mu_{M, n}\right)},
\end{aligned}
$$

where $\tilde{F}_{j, M, n, r} \in L^{\infty}\left(\mu_{M, n}\right)$ are bounded by 1 and $\tilde{k}_{j}$ are integers for $j=1, \ldots, s-2$.

Applying the induction hypothesis for the sequences $S_{r} a \cdot \bar{a}, r \in \mathbb{N}$ (which also admit correlations for Cesàro averages on $\mathbf{I}$ and are bounded by 1 ), the functions $\tilde{F}_{j, M, n, r}$, and the integers $\tilde{k}_{j}, j=1, \ldots, s-2$, and averaging over $r \in \mathbb{N}$, we deduce that the last expression is bounded by 16 times

$$
\limsup _{R \rightarrow \infty} \mathbb{E}_{r \in[R]}\left\|S_{r} a \cdot \bar{a}\right\|_{U^{s-1}(\mathbf{I})} \leq\left(\limsup _{R \rightarrow \infty} \mathbb{E}_{r \in[R]}\left\|S_{r} a \cdot \bar{a}\right\|_{U^{s-1}(\mathbf{I})}^{2^{s-1}}\right)^{\frac{1}{2^{s-1}}}=\|a\|_{U^{s}(\mathbf{I})}^{2} .
$$

Taking square roots, we deduce that (16) holds, completing the induction.

Hence, it suffices to consider the case where $\Phi$ is a non-trivial vertical nilcharacter of $X$, and we have thus reduced matters to proving the following result (note that strong aperiodicity and ergodicity are no longer needed):

Proposition 5.3. Let $s \geq 2$ and $f \in \mathcal{M}$ be a multiplicative function. Let $X=G / \Gamma$ be an s-step nilmanifold, $b \in G$ be an ergodic nilrotation, and $\Phi: X \rightarrow \mathbb{C}$ be a non-trivial vertical nilcharacter. Then for every $(s-1)$-step nilmanifold $Y$ we have

$$
\lim _{M \rightarrow \infty} \limsup _{N \rightarrow \infty} \mathbb{E}_{n \in[N]} \sup _{\psi \in \Psi_{Y}}\left|\mathbb{E}_{m \in[n, n+M]} f(m) \Phi\left(b^{m} \cdot e_{X}\right) \psi(m)\right|=0 .
$$

Remarks. - Note that in this statement we do not impose any assumption on $f \in \mathcal{M}$.

- If $(a(M, n))_{M, n \in \mathbb{N}}$ is bounded and $\lim _{M \rightarrow \infty} \lim \sup _{N \rightarrow \infty} \mathbb{E}_{n \in[N]}|a(M, n)|=0$, we then have that $\lim _{M \rightarrow \infty} \limsup _{N \rightarrow \infty} \mathbb{E}_{n \in[N]}^{\log }|a(M, n)|=0$. It follows that Proposition 5.3 also holds with the averages $\mathbb{E}_{n \in[N]}^{\log }$ in place of the averages $\mathbb{E}_{n \in[N]}$.

\subsection{Step 4 (Disjointifying the intervals $[n, n+M]$ )}

Suppose that Proposition 5.3 fails. Then there exist a multiplicative function $f \in \mathcal{M}$ and

- $\varepsilon>0$

- strictly increasing sequences of integers $\left(M_{k}\right)$ and $\left(N_{k}\right)$ satisfying $M_{k} / N_{k} \rightarrow 0$ as $k \rightarrow \infty$;

- an $s$-step nilmanifold $X=G / \Gamma$ and an ergodic nilrotation $b \in G$;

- a non-trivial vertical nilcharacter $\Phi$ of $X$;

- an $(s-1)$-step nilmanifold $Y$ and $(s-1)$-step nilsequences $\psi_{k, n} \in \Psi_{Y}, k, n \in \mathbb{N}$; 
such that

$$
\mathbb{E}_{n \in\left[N_{k}\right]}\left|\mathbb{E}_{m \in\left[n, n+M_{k}\right)} f(m) \phi(m) \psi_{k, n}(m)\right|>\varepsilon
$$

for all large enough $k \in \mathbb{N}$, where

$$
\phi(m):=\Phi\left(b^{m} \cdot e_{X}\right), \quad m \in \mathbb{N}
$$

We follow the argument used in the proof of [2, Theorem 4] in order to disjointify the intervals $[n, n+M]$. Since $M_{k} / N_{k} \rightarrow 0$, we have for every bounded sequence $(a(k, n))_{k, n \in \mathbb{N}}$ that

$$
\lim _{k \rightarrow \infty}\left(\mathbb{E}_{n \in\left[N_{k}\right]} a(k, n)-\mathbb{E}_{r \in\left[M_{k}\right]} \mathbb{E}_{n \in\left[N_{k}\right]: n \equiv r\left(\bmod M_{k}\right)} a(k, n)\right)=0 .
$$

Applying this for the sequence

$$
a(k, n):=\left|\mathbb{E}_{m \in\left[n, n+M_{k}\right)} f(m) \phi(m) \psi_{k, n}(m)\right|, \quad k, n \in \mathbb{N},
$$

we deduce that for every large enough $k \in \mathbb{N}$ there exists $r_{k} \in\left[M_{k}\right]$ such that

$$
\mathbb{E}_{n \in\left[N_{k}\right]: n \equiv r_{k}\left(\bmod M_{k}\right)}\left|\mathbb{E}_{m \in\left[n, n+M_{k}\right)} f(m) \phi(m) \psi_{k, n}(m)\right|>\varepsilon .
$$

Upon changing the sequences $\left(\psi_{k, n}(m)\right)_{m \in \mathbb{N}}$ by multiplicative constants of modulus 1 that depend on $k$ and $n$ only, we can remove the norm in the previous estimate. Hence, without loss of generality, we can assume for all large enough $k \in \mathbb{N}$ that

$$
\mathbb{E}_{n \in\left[N_{k}\right]: n \equiv r_{k}\left(\bmod M_{k}\right)}\left(\mathbb{E}_{m \in\left[n, n+M_{k}\right)} f(m) \phi(m) \psi_{k, n}(m)\right)>\boldsymbol{\varepsilon} .
$$

Since $M_{k} / N_{k} \rightarrow 0$, we deduce from the last estimate that

$$
\mathbb{E}_{n \in\left[N_{k}\right]} f(n) g_{k}(n)>\varepsilon
$$

for all large enough $k \in \mathbb{N}$, where

$$
g_{k}(n):=\sum_{\ell=1}^{\infty} \mathbf{1}_{\left[(\ell-1) M_{k}, \ell M_{k}\right)}(n) \phi(n) \psi_{k, \ell}(n), \quad k, n \in \mathbb{N}
$$

for some $(s-1)$-step nilsequences $\psi_{k, \ell} \in \Psi_{Y}, k, \ell \in \mathbb{N}$.

Hence, in order to get a contradiction and complete the proof of Proposition 5.3, it remains to verify that

$$
\lim _{k \rightarrow \infty} \mathbb{E}_{n \in\left[N_{k}\right]} f(n) g_{k}(n)=0 .
$$

The only property to be used for the intervals $\left[(\ell-1) M_{k}, \ell M_{k}\right)$ is that their lengths tend to infinity as $k \rightarrow \infty$ uniformly in $\ell \in \mathbb{N}$. 


\section{NIKOS FRANTZIKINAKIS}

\subsection{Step 5 (Applying an orthogonality criterion)}

We will use the following orthogonality criterion for multiplicative functions in $\mathcal{M}$ :

Lemma 5.4 (Kátai [30], see also [8, 37]). For every $\varepsilon>0$ there exist $\delta=\delta(\varepsilon)>0$ and $K:=K(\varepsilon)$ such that the following holds: If $N \geq K$ and $a:[N] \rightarrow \mathbb{C}$ is a finite sequence that is bounded by 1 and satisfies

$$
\max _{\substack{p, q \text { primes } \\ 1<p<q<K}}\left|\mathbb{E}_{n \in[\lfloor N / q]]} a(p n) \overline{a(q n)}\right|<\delta,
$$

then

$$
\sup _{f \in \mathcal{M}}\left|\mathbb{E}_{n \in[N]} f(n) a(n)\right|<\varepsilon .
$$

Remark. Using Vinogradov's bilinear method one can obtain quantitatively superior refinements, see for example [4, Theorem 2].

We deduce from this lemma the following:

Lemma 5.5. Let $N_{k} \rightarrow \infty$ be integers and $a_{k}:\left[N_{k}\right] \rightarrow \mathbb{C}, k \in \mathbb{N}$, be finite sequences that are bounded by 1 and satisfy

$$
\lim _{k \rightarrow \infty} \mathbb{E}_{n \in\left[c N_{k}\right]} a_{k}(p n) \overline{a_{k}\left(p^{\prime} n\right)}=0
$$

for every $p, p^{\prime} \in \mathbb{N}$ with $p \neq p^{\prime}$ and every $c>0$. Then

$$
\lim _{k \rightarrow \infty} \sup _{f \in \mathcal{M}}\left|\mathbb{E}_{n \in\left[N_{k}\right]} f(n) a_{k}(n)\right|=0
$$

Applying Lemma 5.5, we get that in order to prove (18) and complete the proof of Proposition 5.3, it suffices to show that for every $p, p^{\prime} \in \mathbb{N}$ with $p \neq p^{\prime}$ and every $c>0$, we have

$$
\lim _{k \rightarrow \infty} \mathbb{E}_{n \in\left[c N_{k}\right]} g_{k}(p n) \overline{g_{k}\left(p^{\prime} n\right)}=0
$$

where $g_{k}$ is as in (17). Equivalently, we have to show that (the sum below is finite)

$$
\lim _{k \rightarrow \infty} \mathbb{E}_{n \in\left[c N_{k}\right]}\left(\sum_{\ell, \ell^{\prime} \in \mathbb{N}} \mathbf{1}_{I_{k, \ell, \ell^{\prime}}}(n) \phi(p n) \psi_{k, \ell}(p n) \overline{\phi\left(p^{\prime} n\right)} \overline{\psi_{k, \ell^{\prime}}\left(p^{\prime} n\right)}\right)=0
$$

where

$$
I_{k, \ell, \ell^{\prime}}:=\left[\frac{(\ell-1) M_{k}}{p}, \frac{\ell M_{k}}{p}\right) \bigcap\left[\frac{\left(\ell^{\prime}-1\right) M_{k}}{p^{\prime}}, \frac{\ell^{\prime} M_{k}}{p^{\prime}}\right), \quad k, \ell, \ell^{\prime} \in \mathbb{N} .
$$

Note that for fixed $k \in \mathbb{N}$ the intervals $I_{k, \ell, \ell^{\prime}}, \ell, \ell^{\prime} \in \mathbb{N}$, are disjoint (and some of them empty). Since $M_{k} \rightarrow$ $\infty$, they partition the interval $\left[c N_{k}\right]$ into subintervals $J_{k, l}, l=1, \ldots, L_{k}$, with $L_{k} \rightarrow \infty$ and $\min _{l \in\left[L_{k}\right]}\left|J_{k, l}\right| \rightarrow \infty$ as $k \rightarrow \infty$, and a set $Z_{k}$ with $\left|Z_{k}\right| / N_{k} \rightarrow 0$ as $k \rightarrow \infty$. Since $\left|Z_{k}\right| / N_{k} \rightarrow 0$ as $k \rightarrow \infty$, it suffices to show that

$$
\lim _{k \rightarrow \infty} \mathbb{E}_{n \in\left[c N_{k}\right]}\left(\sum_{l \in\left[L_{k}\right]} \mathbf{1}_{J_{k, l}}(n) \phi(p n) \overline{\phi\left(p^{\prime} n\right)} \psi_{k, l}(p n) \overline{\psi_{k, l}^{\prime}\left(p^{\prime} n\right)}\right)=0
$$


where $\psi_{k, l}=\psi_{k, \ell}, \psi_{k, l}^{\prime}=\psi_{k, \ell^{\prime}}$ for some $\ell=\ell(l), \ell^{\prime}=\ell^{\prime}(l)$. Since $\min _{l \in\left[L_{k}\right]}\left|J_{k, l}\right| \rightarrow \infty$ as $k \rightarrow \infty$, it suffices to show that if $J_{k}^{\prime}, k \in \mathbb{N}$, are intervals with $\left|J_{k}^{\prime}\right| \rightarrow \infty$ as $k \rightarrow \infty$, then for every $(s-1)$-step nilmanifold $Y$ we have

$$
\lim _{k \rightarrow \infty} \sup _{\psi \in \Psi_{Y}}\left|\mathbb{E}_{n \in J_{k}^{\prime}} \phi(p n) \overline{\phi\left(p^{\prime} n\right)} \psi(n)\right|=0 .
$$

Thus, in order to complete the proof of Proposition 5.3 it suffices to verify the following asymptotic orthogonality property which has purely dynamical context:

Proposition 5.6. For $s \geq 2$ let $X=G / \Gamma$ be an $s$-step nilmanifold and $b \in G$ be an ergodic nilrotation. Furthermore, let $\Phi, \Phi^{\prime}$ be non-trivial vertical nilcharacters of $X$ with the same frequency. Then for every $p, p^{\prime} \in \mathbb{N}$ with $p \neq p^{\prime}$, every sequence of intervals $\left(I_{N}\right)_{N \in \mathbb{N}}$ with $\left|I_{N}\right| \rightarrow \infty$, and every $(s-1)$-step nilmanifold $Y$, we have

$$
\lim _{N \rightarrow \infty} \sup _{\psi \in \Psi_{Y}}\left|\mathbb{E}_{n \in I_{N}} \Phi\left(b^{p n} \cdot e_{X}\right) \overline{\Phi^{\prime}\left(b^{p^{\prime} n} \cdot e_{X}\right)} \psi(n)\right|=0 .
$$

A model case is when $\Phi\left(b^{n} \cdot e_{X}\right)=\Phi^{\prime}\left(b^{n} \cdot e_{X}\right)=\mathrm{e}\left(n^{s} \beta\right)$ with $\beta$ irrational. Then the statement to be proved reduces to

$$
\lim _{N \rightarrow \infty} \sup _{\psi \in \Psi_{Y}}\left|\mathbb{E}_{n \in I_{N}} \mathrm{e}\left(n^{s} \alpha\right) \psi(n)\right|=0
$$

where $\alpha:=\left(p^{s}-q^{s}\right) \beta$ is irrational. This can be verified easily by using van der Corput's lemma for complex numbers and Lemma 3.3. The proof in the general case is much harder though; it is given in the next subsection.

\subsection{Step 6 (Proof of the dynamical property)}

The goal of this last subsection is to prove Proposition 5.6. Let us remark first that although we were not able to adapt a related argument in [14, Theorem 6.1] to the current setup, we found some of the ideas used there very useful.

The main idea is as follows. We apply the van der Corput lemma for complex numbers $(s-1)$ times in order to cancel out the term $\psi$ (Lemma 3.3 is useful in this regard) and we reduce (19) to verifying $U^{s}(\mathbf{I})$-uniformity for the sequence $\left(\Phi\left(b^{p n} \cdot e_{X}\right) \overline{\Phi^{\prime}\left(b^{p^{\prime} n} \cdot e_{X}\right)}\right)_{n \in \mathbb{N}}$. The fact that the supremum over $\Psi_{Y}$ no longer appears has the additional advantage that we only need to use qualitative (and not quantitative) equidistribution results on nilmanifolds.

The key in obtaining the necessary $U^{s}(\mathbf{I})$-uniformity is to establish that the nilcharacter $\Phi \otimes \Phi^{\prime}$ is non-trivial on the $s$-step nilmanifold $W:=\overline{\left\{\left(b^{p n} \cdot e_{X}, b^{p^{\prime} n} \cdot e_{X}\right), n \in \mathbb{N}\right\}}$. Although the precise structure of the nilmanifold $W$ is very difficult to determine (and depends on the choice of the ergodic nilrotation $b$ ) it is possible to extract partial information on $W$ that suffices for our purposes. This last idea is taken from the proof of [14, Proposition 6.1] and the precise statement is as follows:

Proposition 5.7. For $s \in \mathbb{N}$ let $X=G / \Gamma$ be a connected s-step nilmanifold and $b \in G$ be an ergodic nilrotation. Let $p, p^{\prime} \in \mathbb{N}$ be distinct and let $W$ be the closure of the sequence $\left(b^{p n} \cdot e_{X}, b^{p^{\prime} n} \cdot e_{X}\right)_{n \in \mathbb{N}}$ in $X \times X$. Then $W$ is a nilmanifold that can be represented as $W=H / \Delta$ where $\Delta=\Gamma \times \Gamma$ and $H$ is a subgroup of $G \times G$ such that $\left(b^{p}, b^{p^{\prime}}\right) \in H$ and

$$
\left(u^{p^{s}}, u^{p^{\prime s}}\right) \in H_{s} \quad \text { for every } u \in G_{s} .
$$




\section{NIKOS FRANTZIKINAKIS}

Proof. As remarked in Section 3.2, $W$ is a sub-nilmanifold of $X \times X$. Let

$$
H:=\overline{\left\langle\Gamma \times \Gamma,\left(b^{p}, b^{p^{\prime}}\right)\right\rangle},
$$

that is, $H$ is the smallest closed subgroup of $G \times G$ that contains $\Gamma \times \Gamma$ and $\left(b^{p}, b^{p^{\prime}}\right)$.

Claim. We have that $W=H /(\Gamma \times \Gamma)$.

Indeed, by the definition of $H$ and $W$, we have $H \cdot\left(e_{X}, e_{X}\right) \supset W$. Furthermore, as remarked in Section 3.2, we have $W=H_{1} \cdot\left(e_{X}, e_{X}\right)$ for some closed subgroup $H_{1}$ of $G \times G$ containing the element $\left(b^{p}, b^{p^{\prime}}\right)$. Since $W$ is compact, the set $H_{2}:=H_{1} \cdot(\Gamma \times \Gamma)$ is closed in $G$ (see remarks in Section 3.1). Since $H_{2}$ is a closed subgroup that contains $\left(b^{p}, b^{p^{\prime}}\right)$ and $\Gamma \times \Gamma$, we have $H_{2} \supset H$, hence $H \cdot\left(e_{X}, e_{X}\right) \subset$ $H_{2} \cdot\left(e_{X}, e_{X}\right)=H_{1} \cdot\left(e_{X}, e_{X}\right)=W$. Therefore, $H \cdot\left(e_{X}, e_{X}\right)=W$, which implies that $W=H /(\Gamma \times \Gamma)$. This proves the claim.

It remains to show (20). To this end, it suffices to verify the following:

Claim. Let $j \in\{1, \ldots, s\}$ and $g \in G_{j}$. Then $\left(g^{p^{j}}, g^{p^{\prime j}}\right) \in H_{j} \cdot\left(G_{j+1} \times G_{j+1}\right)$.

We proceed by induction on $j$. We first prove the claim for $j=1$. Let $Z:=G /\left(G_{2} \Gamma\right)$ and $\pi: G \rightarrow Z$ be the natural projection. Since $X$ is connected, the nilmanifold $Z$ is connected. It is also a compact Abelian group, hence $Z$ is a torus. The ergodic nilrotation $b$ projects to the element $\beta:=\pi(b)$ and every power of $\beta$ acts ergodically on the torus $Z$. Since $(\pi \times \pi)(H)$ is the closure of the sequence $\left(\beta^{p n}, \beta^{p^{\prime} n}\right)$ in $Z \times Z$, it follows that $(\pi \times \pi)(H)=\left\{\left(z^{p}, z^{p^{\prime}}\right), z \in Z\right\}$. We deduce from this (recall also that $\left.\Gamma \times \Gamma \subset H\right)$ that

$$
\left\{\left(g^{p}, g^{p^{\prime}}\right), g \in G\right\} \subset H \cdot\left(G_{2} \times G_{2}\right),
$$

which proves the claim for $j=1$.

Let now $j \geq 2$ and suppose that the claim holds for $j-1$, we will show that it holds for $j$. To this end, notice first that if $g, g^{\prime} \in G_{j}$ and $u_{1}, u_{1}^{\prime}, u_{2}, u_{2}^{\prime} \in G_{j+1}$, then

$$
g^{p^{j}} u_{1} \cdot g^{\prime p^{j}} u_{1}^{\prime}=\left(g g^{\prime}\right)^{p^{j}} \bmod G_{j+1}, \quad g^{p^{\prime j}} u_{2} \cdot g^{\prime p^{\prime j}} u_{2}^{\prime}=\left(g g^{\prime}\right)^{p^{j}} \bmod G_{j+1} .
$$

It follows that the family of elements $g$ of $G_{j}$ for which the conclusion of the claim holds is a subgroup of $G_{j}$. Therefore, it suffices to prove that the conclusion of the claim holds when $g$ is a commutator, that is, $g=[h, v]$ for some $h \in G$ and some $v \in G_{j-1}$. By the $j=1$ case of the claim there exist $u, u^{\prime} \in G_{2}$ such that $\left(h^{p} u, h^{p^{\prime}} u^{\prime}\right) \in H$ and by the induction hypothesis there exist $w, w^{\prime} \in G_{j}$ such that $\left(v^{p^{j-1}} w, v^{p^{\prime j-1}} w^{\prime}\right) \in H_{j-1}$. Then the commutator $\left(\left[h^{p} u, v^{p^{j-1}} w\right],\left[h^{p^{\prime}} u^{\prime}, v^{p^{j-1}} w^{\prime}\right]\right)$ of these elements belongs to $H_{j}$. Since

$$
\left[h^{p} u, v^{p^{j-1}} w\right]=[h, v]^{p^{j}}=g^{p^{j}} \bmod G_{j+1}, \quad\left[h^{p^{\prime}} u^{\prime}, v^{p^{\prime j-1}} w^{\prime}\right]=[h, v]^{p^{\prime j}}=g^{p^{\prime j}} \bmod G_{j+1},
$$

we deduce that

$$
\left(g^{p^{j}}, g^{p^{\prime j}}\right) \in H_{j} \cdot\left(G_{j+1} \times G_{j+1}\right) .
$$

This completes the proof of the claim.

Setting $j=s$ and using that $G_{s+1}$ is trivial we deduce (20), completing the proof. 


\section{ERgodicity of The Liouville System Implies the Chowla Conjecture}

Lemma 5.8. For $s \geq 2$ let $W=H / \Delta$ be an s-step nilmanifold and $h \in H$ be an ergodic nilrotation. Let $\Phi$ be a non-trivial vertical nilcharacter of $W$ and

$$
\phi(n):=\Phi\left(h^{n} \cdot e_{W}\right), \quad n \in \mathbb{N} .
$$

Then $\|\phi\|_{U^{s}(\mathbf{I})}=0$ for every sequence of intervals $\mathbf{I}=\left(I_{N}\right)_{N \in \mathbb{N}}$ with $\left|I_{N}\right| \rightarrow \infty$.

Proof. As remarked in Section 3.2, we have $\|\phi\|_{U^{s}(\mathbf{I})}=\|\Phi\|_{s}$, where the seminorm is computed for the system induced on $W$ with the normalized Haar measure $m_{W}$ by the ergodic nilrotation by $h \in H$. Let $Z_{s-1}(W)$ be defined as in Section 2.4.

It is implicit in [28, Theorem 13.1] and also follows by combining [44, Lemma 4.5] and [31], that $L^{2}\left(Z_{s-1}(W)\right)$ consists exactly of those functions in $L^{2}\left(m_{W}\right)$ that are $H_{s}$-invariant ([31] shows that the factors $z_{s}$ and $Y_{s}$ defined in [28] and [44] respectively are the same). Since $\Phi$ is a non-trivial vertical nilcharacter of $W$, it is orthogonal to any $H_{s}$-invariant function in $L^{2}\left(m_{W}\right)$, hence $\Phi$ is orthogonal to any function in $L^{2}\left(z_{s-1}(W)\right)$. As remarked in Section 2.4, this implies that $\|\Phi\|_{s}=0$ and completes the proof.

Proposition 5.9. For $s \geq 2$ let $W=H / \Delta$ be an s-step nilmanifold and $h \in H$ be an ergodic nilrotation. Furthermore, let $\Phi$ be a non-trivial vertical nilcharacter of $W$. Then for every sequence of intervals $\mathbf{I}=\left(I_{N}\right)_{N \in \mathbb{N}}$ with $\left|I_{N}\right| \rightarrow \infty$ and every $(s-1)$-step nilmanifold $Y$ we have

$$
\lim _{N \rightarrow \infty} \sup _{\psi \in \Psi_{Y}}\left|\mathbb{E}_{n \in I_{N}} \Phi\left(h^{n} \cdot e_{W}\right) \psi(n)\right|=0 .
$$

Proof. Let $\phi(n):=\Phi\left(h^{n} \cdot e_{W}\right), n \in \mathbb{N}$. By Lemma 5.8 we have that $\|\phi\|_{U^{s}(\mathbf{I})}=0$.

It follows from Lemma 3.3 that it suffices to establish the following: Let $s \in \mathbb{N}, \mathbf{I}=\left(I_{N}\right)_{N \in \mathbb{N}}$ be a sequence of intervals with $\left|I_{N}\right| \rightarrow \infty$, and $a \in \ell^{\infty}(\mathbb{N})$ be a sequence that admits correlations for Cesàro averages on $\mathbf{I}$. Furthermore, for $N \in \mathbb{N}$, let $\left(X_{N}, X_{N}, \mu_{N}, T_{N}\right)$ be a system, $F_{0, N}, \ldots, F_{s-1, N} \in L^{\infty}\left(\mu_{N}\right)$ be functions bounded by 1 , and let $k_{1}, \ldots, k_{s-1} \in \mathbb{Z}$. Then we have

$$
\limsup _{N \rightarrow \infty}\left|\mathbb{E}_{n \in I_{N}} a(n) \int F_{0, N} \cdot T_{N}^{k_{1} n} F_{1, N} \cdot \ldots \cdot T_{N}^{k_{s-1} n} F_{s-1, N} d \mu_{N}\right| \leq 4\|a\|_{U^{s}(\mathbf{I})} .
$$

This estimate can be proved by induction on $s$ in a rather standard way using the van der Corput lemma for inner product spaces, the details are given in [12, Section 2.3.1].

We are now ready to prove Proposition 5.6 which is the last step in the proof of Theorem 1.6.

Proof of Proposition 5.6. We argue by contradiction. Suppose that for some $s \geq 2$ there exist an $s$-step nilmanifold $X=G / \Gamma$, an ergodic nilrotation $b \in G$, non-trivial vertical nilcharacters $\Phi, \Phi^{\prime}$ of $X$ with the same frequency, $p, p^{\prime} \in \mathbb{N}$ with $p \neq p^{\prime}$, a sequence of intervals $\left(I_{N}\right)_{N \in \mathbb{N}}$ with $\left|I_{N}\right| \rightarrow \infty$, and an $(s-1)$-step nilmanifold $Y$, such that

$$
\limsup _{N \rightarrow \infty} \sup _{\psi \in \Psi_{Y}}\left|\mathbb{E}_{n \in I_{N}} \Phi\left(b^{p n} \cdot e_{X}\right) \overline{\Phi^{\prime}\left(b^{p^{\prime} n} \cdot e_{X}\right)} \psi(n)\right|>0
$$




\section{NIKOS FRANTZIKINAKIS}

We first reduce matters to the case where the nilmanifold $X$ is connected. As remarked in Section 3.2, there exists $r \in \mathbb{N}$ such that $b^{r}$ acts ergodically on the connected component $X_{0}$ of the nilmanifold $X$. Then for some $j \in\{0, \ldots, r-1\}$ we have

$$
\limsup _{N \rightarrow \infty} \sup _{\psi \in \Psi_{Y}}\left|\mathbb{E}_{n \in I_{N}} \Phi\left(b^{p(r n+j)} \cdot e_{X}\right) \overline{\Phi^{\prime}\left(b^{p^{\prime}(r n+j)} \cdot e_{X}\right)} \psi(r n+j)\right|>0 .
$$

Since $(\psi(r n+j))_{n \in \mathbb{N}} \in \Psi_{Y}$ for every $r, s \in \mathbb{N}$, we deduce that

$$
\underset{N \rightarrow \infty}{\limsup } \sup _{\psi \in \Psi_{Y}}\left|\mathbb{E}_{n \in I_{N}} \tilde{\Phi}\left(\tilde{b}^{p n} \cdot e_{X}\right) \overline{\tilde{\Phi}^{\prime}\left(\tilde{b}^{p^{\prime} n} \cdot e_{X}\right)} \psi(n)\right|>0,
$$

where $\tilde{b}:=b^{r}$ is an ergodic nilrotation of $X_{0}$, and the functions $\tilde{\Phi}, \tilde{\Phi}^{\prime}: X_{0} \rightarrow \mathbb{C}$, defined by $\tilde{\Phi}(x):=$ $\Phi\left(b^{p j} x\right), \tilde{\Phi}^{\prime}(x):=\Phi\left(b^{p^{\prime} j} x\right), x \in X_{0}$, are non-trivial vertical nilcharacters of $X_{0}$ (see the discussion in Section 3.3) with the same frequency. Hence, we can assume that the nilmanifold $X$ is connected.

Let $h:=\left(b^{p}, b^{\prime p^{\prime}}\right)$. By the discussion in Section 3.2 and Proposition 5.7, the element $h$ acts ergodically on a nilmanifold $W$ that can be represented as $W=H / \Delta$ where $H$ is a subgroup of $G \times G$ such that $h \in H$ and $\left(u^{p^{s}}, u^{p^{\prime s}}\right) \in H_{s}$ for every $u \in G_{s}$.

We will show that the restriction of the function $\Phi \otimes \overline{\Phi^{\prime}}$ on $W$ is a non-trivial vertical nilcharacter of $W$. To this end, we use our hypothesis that

$$
\Phi(u \cdot x)=\chi(u) \Phi(x) \text { and } \Phi^{\prime}(u \cdot x)=\chi(u) \Phi^{\prime}(x) \text { for } u \in G_{s} \text { and } x \in X,
$$

where $\chi$ is a non-trivial element of the dual of $G_{s}$ that is $\left(G_{s} \cap \Gamma\right)$-invariant. Hence,

$$
\left(\Phi \otimes \overline{\Phi^{\prime}}\right)\left(\left(u, u^{\prime}\right) \cdot\left(x, x^{\prime}\right)\right)=\chi(u) \bar{\chi}\left(u^{\prime}\right)\left(\Phi \otimes \overline{\Phi^{\prime}}\right)\left(x, x^{\prime}\right), \quad \text { for } u, u^{\prime} \in G_{s} \text { and } x, x^{\prime} \in X .
$$

Since $H_{s} \subset G_{s} \times G_{s}$, it follows from this identity that $\Phi \otimes \overline{\Phi^{\prime}}$ is a vertical nilcharacter of $W=H / \Delta$. It remains to show that $\chi \cdot \bar{\chi}$ is non-trivial on $H_{s}$. Arguing by contradiction, suppose it is. Since $\left(u^{p^{s}}, u^{p^{\prime s}}\right) \in H_{s}$ for every $u \in G_{s}$, we get

$$
\chi\left(u^{p^{s}-p^{\prime s}}\right)=\chi\left(u^{p^{s}}\right) \overline{\chi\left(u^{p^{\prime s}}\right)}=1 \quad \text { for every } u \in G_{s} .
$$

Since $G_{s}$ is connected for $s \geq 2$ and $p \neq p^{\prime}$, the map $u \mapsto u^{p^{s}-p^{\prime s}}$ is onto $G_{s}$, hence $\chi$ is the trivial character on $G_{s}$, a contradiction.

Combining the above, we get that Proposition 5.9 applies and gives

$$
\lim _{N \rightarrow \infty} \sup _{\psi \in \Psi_{Y}}\left|\mathbb{E}_{n \in I_{N}}\left(\Phi \otimes \overline{\Phi^{\prime}}\right)\left(h^{n} \cdot e_{W}\right) \psi(n)\right|=0 .
$$

This contradicts (21) and completes the proof of Proposition 5.6.

\section{References}

[1] H. el Abdalaoui, J. Kułaga-Przymus, M. Lemańczyk, T. de la Rue. The Chowla and the Sarnak conjectures from ergodic theory point of view. Discrete Contin. Dyn. Syst. 37 (2017), no. 6, 2899-2944. 2 


\section{ERgodicity of The Liouville System Implies the ChOWla Conjecture}

[2] H. el Abdalaoui, M. Lemańczyk, T. Rue. Automorphisms with quasi-discrete spectrum, multiplicative functions and average orthogonality along short intervals. Int. Math. Res. Not., Volume 2017, Issue 14, (2017), 4350-4368 33

[3] V. Bergelson, B. Host, B. Kra, with an appendix by I. Ruzsa. Multiple recurrence and nilsequences. Inventiones Math. 160 (2005), no. 2, 261-303. 16, 17

[4] J. Bourgain, P. Sarnak, T. Ziegler. Disjointness of Möbius from horocycle flows. From Fourier analysis and number theory to Radon transforms and geometry. Dev. Math. 28, Springer, New York, (2013), 67-83. 34

[5] S. Chowla. The Riemann Hypothesis and Hilberts Tenth Problem. Mathematics and Its Applications 4, Gordon and Breach Science Publishers, New York, 1965. 1

[6] Q. Chu, N. Franzikinakis. Pointwise convergence for cubic and polynomial ergodic averages of non-commuting transformations. Ergodic Theory Dynam. Systems 32 (2012), 877-897. 11

[7] Q. Chu, N. Franzikinakis, B. Host. Ergodic averages of commuting transformations with distinct degree polynomial iterates. Proc. Lond. Math. Soc. 102 (2011), 801-842. 23

[8] H. Daboussi. Fonctions multiplicatives presque périodiques B. D'après un travail commun avec H. Delange. Journées Arithmétiques de Bordeaux (Conf., Univ. Bordeaux, Bordeaux, 1974), pp. 321-324. Asterisque 24-25 (1975), 321-324. 34

[9] P. Elliott. Multiplicative functions $|g| \leq 1$ and their convolutions: An overview. Séminaire de Théorie des Nombres, Paris 1987-88. Progress in Mathematics 81 (1990), 63-75. 4

[10] P. Elliott. On the correlation of multiplicative and the sum of additive arithmetic functions. Mem. Amer. Math. Soc. 112 (1994), no. 538, viii+88pp. 4

[11] L. Flaminio, K. Fraczek, J. Kułaga-Przymus, M. Lemańczyk. Approximate orthogonality of powers for ergodic affine unipotent diffeomorphisms on nilmanifolds. (2016), arXiv:1609.00699. 30

[12] N. Frantzikinakis. Multiple correlation sequences and nilsequences. Invent. Math. 202 (2015), no. 2, 875-892. 18, 37

[13] N. Frantzikinakis. An averaged Chowla and Elliott conjecture along independent polynomials. To appear in Int. Math. Res. Not. arXiv:1606.08420. 13, 15

[14] N. Frantzikinakis, B. Host. Higher order Fourier analysis of multiplicative functions and applications. J. Amer. Math. Soc. 30 (2017), 67-157. 5, 11, 25, 29, 30, 35

[15] N. Frantzikinakis, B. Host. The logarithmic Sarnak conjecture for ergodic weights. (2017), arXiv: $1708.00677 .4,14$

[16] N. Frantzikinakis, B. Host, B. Kra. The polynomial multidimensional Szemerédi theorem along shifted primes. Isr. J. Math. 194 (2013), 331-348. 15 


\section{NIKOS FRANTZIKINAKIS}

[17] H. Furstenberg. Ergodic behavior of diagonal measures and a theorem of Szemerédi on arithmetic progressions. J. Analyse Math. 31 (1977), 204-256. 8

[18] H. Furstenberg. Recurrence in ergodic theory and combinatorial number theory. Princeton University Press, Princeton, 1981. 2

[19] A. Gomilko, D. Kwietniak, M. Lemańczyk. Sarnak's conjecture implies the Chowla conjecture along a subsequence. (2017), arXiv:1710.07049 4

[20] W. Gowers. A new proof of Szemerédi's theorem. Geom. Funct. Anal. 11 (2001), 465-588. 11, 21

[21] A. Granville, K. Soundararajan. Multiplicative Number Theory: The pretentious approach. Book manuscript in preparation. 12

[22] B. Green, T. Tao. An arithmetic regularity lemma, associated counting lemma, and applications. An irregular mind, Bolyai, Soc. Math. Stud. 21, János Bolyai Math. Soc., Budapest, (2010), 261-334. 24,25

[23] B. Green, T. Tao. Linear equations in the primes. Ann. of Math. 171 (2010), 1753-1850. 5, 15

[24] B. Green, T. Tao. The quantitative behaviour of polynomial orbits on nilmanifolds. Ann. of Math. 175 (2012), 465-540. 5, 15, 17, 19

[25] B. Green, T. Tao. The Möbius function is strongly orthogonal to nilsequences. Ann. of Math. 175 (2012), no. 2, 541-566. 5, 30

[26] B. Green, T. Tao, T. Ziegler. An inverse theorem for the Gowers $U^{s+1}[N]$-norm. Ann. of Math. 176 (2012), no. 2, 1231-1372. 3, 5, 15, 24, 28

[27] G. Halász. Über die Mittelwerte multiplikativer zahlentheoretischer Funktionen. Acta Math. Acad. Sci. Hung. 19 (1968), 365-403. 13

[28] B. Host, B. Kra. Non-conventional ergodic averages and nilmanifolds. Ann. of Math. 161 (2005), 397-488. 3, 9, 10, 24, 37

[29] B. Host, B. Kra. Uniformity seminorms on $l^{\infty}$ and applications. J. Analyse Math. 108 (2009), 219-276. 10

[30] I. Kátai. A remark on a theorem of H. Daboussi. Acta Math. Hungar. 47 (1986), 223-225. 34

[31] A. Leibman. Host-Kra and Ziegler factors and convergence of multiple averages. Handbook of Dynamical Systems, vol. 1B, B. Hasselblatt and A. Katok, eds., Elsevier (2005), 841-853 15, 37

[32] A. Leibman. Pointwise convergence of ergodic averages for polynomial sequences of rotations of a nilmanifold. Ergodic Theory Dynam. Systems 25 (2005), no. 1, 201-213. 16, 17

[33] E. Lesigne. Sur une nil-variété, les partiesminimales associées à une translation sont uniquement ergodiques. Ergodic Theory Dynam. Systems 11 (1991), no. 2, 379-391. 17 
[34] K. Matomäki, M. Radziwiłł. Multiplicative functions in short intervals. Ann. of Math. 183 (2016), 1015-1056. 2, 13

[35] K. Matomäki, M. Radziwiłł, T. Tao. An averaged form of Chowla's conjecture. Algebra \& Number Theory 9 (2015), 2167-2196. 2, 4, 12, 13, 29

[36] K. Matomäki, M. Radziwiłł, T. Tao. Sign patterns of the Liouville and Möbius functions. Forum Math. Sigma 4 (2016). 2

[37] H. Montgomery, R. Vaughan. Exponential sums with multiplicative coefficients. Invent. Math. 43 (1977), no. 1, 69-82. 34

[38] P. Sarnak. Three lectures on the Möbius function randomness and dynamics. Manuscript (2010). http://publications.ias.edu/sarnak/paper/506. 2, 3

[39] P. Sarnak. Möbius randomness and dynamics. Not. S. Afr. Math. Soc. 43 (2012), no. 2, 89-97. 3

[40] T. Tao. Higher order Fourier analysis. Graduate studies in mathematics 142, American Mathematical Society, (2012). 19

[41] T. Tao. The logarithmically averaged Chowla and Elliott conjectures for two-point correlations. Forum Math. Pi 4 (2016). 2, 5, 14, 17

[42] T. Tao. Equivalence of the logarithmically averaged Chowla and Sarnak conjectures. In: C. Elsholtz, P. Grabner, Number Theory - Diophantine Problems, Uniform Distribution and Applications. Springer, Cham, (2017), 391-421. 5, 11, 19

[43] T. Tao, J. Teräväinen. The structure of logarithmically averaged correlations of multiplicative functions, with applications to the Chowla and Elliott conjectures. (2017), arXiv:1708.02610v1. $2,14,28$

[44] T. Ziegler. Universal characteristic factors and Furstenberg averages. J. Amer. Math. Soc. 20 (2007), 53-97. 37

\section{AUTHOR}

Nikos Frantzikinakis

Department of Mathematics and Applied Mathematics

University of Crete

Voutes University Campus, Heraklion 70013, Greece

frantzikinakis [at] gmail [dot] com

http://users.math.uoc.gr/ nikosf/ 